\title{
The last vestiges of naturalness
}

\author{
Asimina Arvanitaki, ${ }^{a}$ Masha Baryakhtar, ${ }^{a}$ Xinlu Huang, ${ }^{a}$ Ken Van Tilburg ${ }^{a}$ and \\ Giovanni Villadoro ${ }^{a, b}$ \\ ${ }^{a}$ Stanford Institute for Theoretical Physics, Department of Physics, \\ Stanford University, Stanford, CA 94305, U.S.A. \\ ${ }^{b}$ Abdus Salam International Centre for Theoretical Physics \\ Strada Costiera 11, 34151, Trieste, Italy \\ E-mail: aarvan@stanford.edu, mbaryakh@stanford.edu, \\ xinluh@stanford.edu, kenvt@stanford.edu, giovanni.villadoro@ictp.it
}

ABSTRACT: Direct LHC bounds on colored SUSY particles now corner naturalness more than the measured value of the Higgs mass does. Bounds on the gluino are of particular importance, since it radiatively "sucks" up the stop and Higgs-up soft masses. As a result, even models that easily accommodate a $125 \mathrm{GeV}$ Higgs are almost as tuned as the simplest version of SUSY, the MSSM: at best at the percent level. In this paper, we further examine how current LHC results constrain naturalness in three classes of models that may relax LHC bounds on sparticles: split families, baryonic RPV, and Dirac gauginos. In models of split families and bRPV, the bounds on the gluino are only slightly reduced, resulting in a few percent tuning. In particular, having a natural spectrum in bRPV models typically implies that tops, $W \mathrm{~s}$, and $Z \mathrm{~s}$ are easily produced in the cascade decays of squarks and gluinos. The resulting leptons and missing energy push the gluino mass limit above $1 \mathrm{TeV}$. Even when the gluino has a Dirac mass and does not contribute to the stop mass at one loop, tuning reappears in calculable models because there is no symmetry imposing the supersoft limit. We conclude that, even if sparticles are found at LHC-14, naturalness will not emerge triumphant.

KeYwords: Beyond Standard Model, Supersymmetric Standard Model

ARXIV EPRINT: 1309.3568 


\section{Contents}

1 Introduction 1

1.1 Quantifying naturalness 2

1.2 Setting the standard for minimal fine-tuning in the MSSM 5

2 Split families $\quad 7$

2.1 Experimental bounds $\quad 7$

2.2 Toy model 8

2.3 Fine tuning 9

3 Baryonic RPV 10

$\begin{array}{lll}3.1 & \text { Constraints on bRPV couplings } & 10\end{array}$

$\begin{array}{lll}3.2 & \text { Toy model } & 13\end{array}$

$\begin{array}{lll}3.3 & \text { Fine tuning } & 14\end{array}$

4 Dirac gauginos $\quad 15$

$\begin{array}{lll}5 & \text { Summary } & 19\end{array}$

A Taking into account correlations between different sources of tuning $\quad 23$

B Split families details $\quad 24$

C RPV details $\quad 25$

\section{Introduction}

The LHC has brought particle physics face to face with data. It discovered the last missing particle of the Standard Model, the Higgs boson, and it is placing increasingly stringent bounds on the new physics proposed to protect its mass. The principle of Naturalness, which has been guiding beyond the Standard Model (BSM) physics for several decades, is now in question, and the possibility that the electroweak (EW) scale is tuned by environmental selection in a multiverse looks increasingly alluring.

Already after the LEP experiments, the absence of any BSM particle at or below the electroweak scale suggested the presence of a small gap between the $Z$ mass and the scale of new physics. In fact, the principle of Naturalness has already failed once: the smallness of the cosmological constant (CC) produces a hierarchy at least 50 orders of magnitude more severe, already a strong indication for environmental selection in our universe.

However, unlike for the CC problem, many dynamical solutions for stabilizing the EW scale exist. Among these, supersymmetry (SUSY) plays a special role because it 
quantitatively predicts the unification of the gauge couplings [1,2]. The latter gives an independent, although weaker, motivation to the presence of new particles around the $\mathrm{TeV}$ scale [3].

It is well-known that in the minimal implementation of SUSY - the MSSM - raising the Higgs mass to its experimental value of $125 \mathrm{GeV}$ requires stop masses above a $\mathrm{TeV}$, already implying a tuning at the percent level or worse (depending on $A$-terms). Such large radiative corrections can be avoided if there are extra contributions to the Higgs quartic self-coupling, as in the NMSSM, in which a singlet provides this contribution. However, direct bounds on colored superpartners from the LHC are already so strong that even after raising the value of the Higgs mass in a natural way, the fine tuning does not improve. Thus, the only hope for finding a natural implementations of SUSY is to look for models that can relax the most stringent bounds set by the LHC searches. Recently, three classes of models have attracted special attention: split families (also dubbed "Natural" SUSY), baryonic R-parity violating SUSY (bRPV), and Dirac gauginos.

The structure of the paper is as follows: in the remainder of this section, we discuss the sources of tuning in SUSY theories after the first run of the LHC. In particular, the MSSM and the NMSSM constitute a benchmark for the other models. In sections 2, 3 and 4, we analyze split-family, bRPV, and Dirac gaugino models, respectively. We discuss experimental constraints and compare the fine tuning in explicit models wherever possible. In section 5, we summarize our conclusions about the various models and assess the status of naturalness in SUSY.

\subsection{Quantifying naturalness}

The level of tuning in a theory can be measured by the sensitivity of the low-energy observables $\mathcal{O}$ to the fundamental UV parameters $a_{i}$; it is commonly quantified by the formula [4]:

$$
\mathrm{FT}_{\mathcal{O}}=\left[\sum_{i}\left(\frac{\partial \log \mathcal{O}}{\partial \log a_{i}}\right)^{2}\right]^{-1 / 2}
$$

The total amount of fine tuning of a theory is given by the product of the fine tunings of each independent low-energy observable,

$$
\mathrm{FT}=\prod_{i} \mathrm{FT}_{\mathcal{O}_{i}}
$$

For theories that try to address the hierarchy problem, the dominant source of fine tuning is usually the one associated to the EW scale. There are cases in which the tuning resides in other observables; we take these into account with eq. (1.2). ${ }^{1}$

In the decoupling limit for the lightest Higgs state $h$, the tree-level effective potential is

$$
V(h)=\frac{1}{2} m_{0}^{2} h^{2}+\frac{1}{4} \lambda h^{4} .
$$

\footnotetext{
${ }^{1}$ When two observables are not independent one should take into account the degree of correlation to avoid overestimating the tuning (details in appendix A).
} 
At the minimum $\langle h\rangle=v$, the physical Higgs mass $m_{h}$, the vev and the Lagrangian mass term $m_{0}$ are related by

$$
m_{h}^{2}=2 \lambda v^{2}=-2 m_{0}^{2} .
$$

It is clear that in this case the tuning for $v^{2}$ and for $m_{h}^{2}$ are equal, and can be computed independently of the quartic coupling and its origin.

In the MSSM at large $\tan \beta$,

$$
m_{h}^{2}=-2 m_{0}^{2}=-2\left(m_{H_{u}}^{2}+|\mu|^{2}\right),
$$

which implies that the higgsino cannot be much heavier than the lightest Higgs without tree-level tuning. From the one loop RGE,

$$
\partial_{t} m_{H_{u}}^{2}=\frac{6\left|y_{t}\right|^{2}}{(4 \pi)^{2}}\left(m_{\tilde{t}_{L}}^{2}+m_{\tilde{t}_{R}}^{2}+\left|A_{t}\right|^{2}\right)+\ldots
$$

we see that $m_{H_{u}}^{2}$ receives large corrections proportional to the stop masses and the $A$-term, which thus need to be small as well. Finally, the stop mass itself is attracted to the gluino mass in the IR,

$$
\partial_{t} m_{\tilde{t}}^{2}=-\frac{8 \alpha_{s}}{3 \pi} M_{3}^{2}+\ldots
$$

which indirectly attracts $m_{H_{u}}^{2}$. Because of the large coefficients in eqs. (1.6) and (1.7) and the strong experimental bounds, the gluino contribution is typically the dominant source of tuning. Note that to capture non-linear effects in the RGEs, we implement a full-scale RG analysis with tuning against UV parameters, instead of just using the leading-log estimates. The magnitude of the RG contributions to the Higgs mass from the stop and the gluino increases with the amount of running; therefore models with a low mediation scale are typically less tuned.

While eq. (1.5) is exactly true in the large- $\tan \beta$ limit of the MSSM, we use the same formula as a reliable estimate at lower values of $\tan \beta$ and in more general Higgs sectors. Relaxing the large-tan $\beta$ assumption does not alleviate the tuning because in the decoupling limit the physical Higgs always couples to the stop with the physical top Yukawa coupling $\left(m_{t} / v\right)$, independently of $\tan \beta .^{2}$ In extended Higgs sectors, the tuning can be reduced by changing the relation between the measured Higgs mass and the vev of eq. (1.5). However, since the $125 \mathrm{GeV}$ resonance couples to vector bosons with very SM-like couplings (up to $\sim 25 \%[5,6]$ ), it should be the one mostly responsible for EW symmetry breaking and thus for most of the top mass. For example, in the MSSM the mixing between the two Higgs doublets is already constrained to be below 10\% [7]. This implies a significant coupling to the stop, which causes the tuning. On the other hand, in the NMSSM it is possible to adjust the parameters to simulate SM couplings for a $125 \mathrm{GeV}$ resonance that is not the state mostly responsible for EWSB. In this case, the tuning of the EW vev is shifted to tuning the values of the Higgs couplings to be SM-like. Large mixings generically imply

\footnotetext{
${ }^{2}$ At $\tan \beta \approx 1$, the tuning is moved from the minimization condition in eq. (1.5) to the requirement $2 B_{\mu} \simeq\left(m_{H_{u}}^{2}+m_{H_{d}}^{2}+2|\mu|^{2}\right)$, again imposing an additive relation on mass parameters.
} 


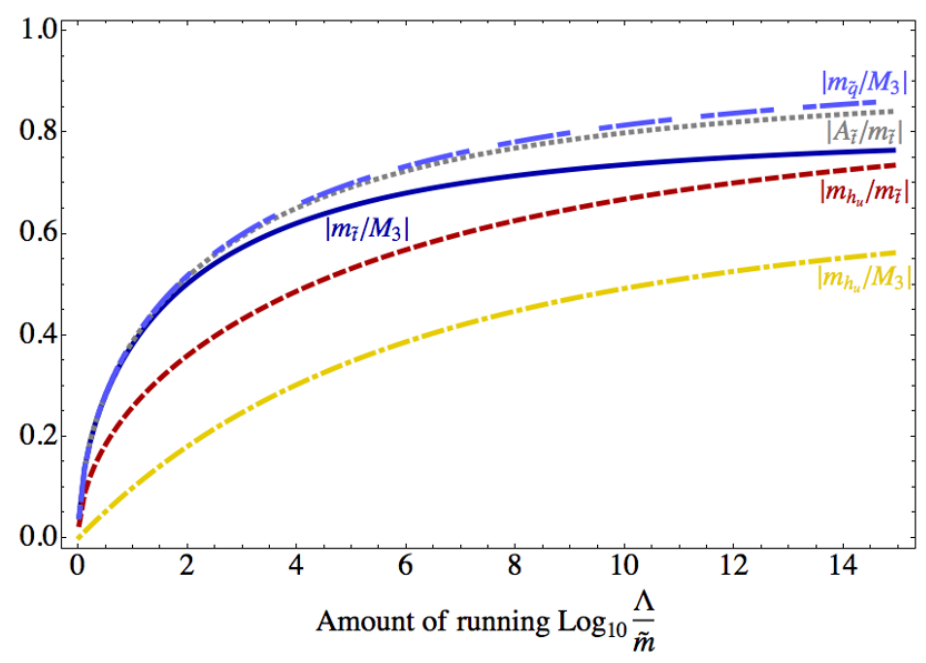

Figure 1. The gluino sucks effect: even starting with vanishing boundary conditions for all scalar soft terms at the scale $\Lambda$, they are quickly generated in the IR by the gluino mass contributions. Already after one decade of running the average stop mass $m_{\tilde{t}}=\sqrt{\left(m_{\tilde{t}_{L}}^{2}+m_{\tilde{t}_{R}}^{2}\right) / 2}$ is almost a factor 2 below the gluino and after three decades also $\left|m_{H_{u}}\right|$ is within a factor of 4 from the gluino. Few decades of running are enough for the soft masses to saturate their IR fixed values.

big deviations of the Higgs couplings from their SM values - a natural Higgs is not the SM Higgs [8].

One may think that a light stop, regardless of the gluino mass, improves the $m_{h}^{2}$ tuning by reducing the one-loop contribution in eq. (1.6). On the contrary, this scenario is even more tuned because there are two unnaturally light scalars. The large coefficient in front of $M_{3}^{2}$ in eq. (1.7) imposes a IR fixed relation between $m_{\tilde{t}}$ and $M_{3}$, and the least tuned spectra are those where $m_{\tilde{t}} \sim M_{3}$. As shown in figure 1 , both $m_{\tilde{t}}$ and $m_{H_{u}}$ are quickly attracted by the gluino even if they vanish at the messenger scale. Deviating from this IR prediction requires more tuning. Models with a low mediation scale are thus preferred.

In order to significantly relax the bounds on Naturalness, one has to alleviate the strong "gluino sucks" effect. We can envision two possible ways to accomplish this: hiding the gluino (by relaxing the collider bounds) or canceling the gluino effects with other big contributions. The first class of solutions includes split family models (which decrease the production cross section) and bRPV models (which hide the collider signatures). The second class includes Dirac gaugino models (where contributions from extra partner fields cancel the gluino ones). All these models, which are discussed in detail later, are based on symmetries or a dynamical structure. Their beneficial properties are robust against the UV details of the theory, thus giving them an opportunity to alleviate the tuning.

The models that may improve naturalness do not include those that suppress the RG effects of the gluino without a symmetry or a robust dynamical mechanism. These include stealth/compressed spectra models where the gluino bounds are relaxed by means of a "cleverly arranged" low-energy spectrum, and focus-point-like models (such as $[9,10]$ ) where the gluino RG contributions are canceled against other enhanced contributions 
(sourced by the other gauginos, squarks, etc.) via accurately chosen boundary conditions at high energies. In all these cases there is no symmetry imposing the UV relations, and the tuning is shifted to the carefully chosen boundary conditions. The presence of these accidental cancellations in the RGE and the reason why they are not a solution of the tuning problem was already explained in the seminal paper by Barbieri and Giudice [4] well before the more recent revival after the LEP, Tevatron and LHC negative results. Also note that such tuning cannot be avoided by replacing the tuned UV parameters with discrete ones. Even if the standard formula in eq. (1.1) does not reflect this kind of tuning, the model building assumptions generate a mini-landscape, where only a small fraction of models satisfy the required UV conditions. In fact, these kinds of models are implementing a solution to the hierarchy problem similar in spirit to the string landscape: after moduli stabilization, string theory vacua are determined by choices of discrete parameters (topology, flux units, number of branes, etc.) and each "model" has no free continuous parameter, thus no tuning according to eq. (1.1). This example highlights the danger of blindly applying tuning formulae such as eq. (1.1) while practicing extreme model building.

In the remainder of this paper, we study low-scale gauge mediated (GMSB) models, which minimize the effects of RG flows, automatically address the flavor problem, preserve unification, and provide a calculable framework for computing the tuning. We minimize the boundary contribution to the scalar masses by taking a large number of messengers and allow extra contributions to the stop and Higgs soft masses at the UV as suggested by models solving the $\mu-B_{\mu}$ problem [11-13]. In computing the tuning according to eqs. (1.1) and (1.2), we use the observables $\mathcal{O}_{i}=\left\{m_{h}^{2}, m_{\tilde{t}}^{2}\right\}$ evaluated at the scale of the stop masses and with $m_{h}^{2}$ as in eq. (1.5). We also use different sets of the UV parameters $a_{i}$, which depend on the explicit model and will be specified in the appropriate sections. This procedure gives us a reliable framework to compare the tuning across models. We expect our explicit models to exhibit nearly optimal tuning.

\subsection{Setting the standard for minimal fine-tuning in the MSSM}

To quantify the status of naturalness in SUSY, we start with its simplest implementation - the MSSM. For small $A$-terms and large $\tan \beta$ the value of the Higgs mass forces the stop masses to be close to $10 \mathrm{TeV}[15,16]$, which implies a tuning of $10^{-3}$. This corresponds to the large $\tan \beta$ tail of the Mini-Split family of models.

The situation improves for large $A$-terms, as the experimental value of the Higgs mass can be achieved with smaller stop masses of $\mathcal{O}(1 \mathrm{TeV})$. In figure $2(\mathrm{a})$, we show tuning contours in the stop-gluino mass plane. We assume universal gaugino and squark boundary conditions, and allow for extra contributions to the stop and Higgs soft masses to deviate from the GMSB prediction and fix $\mu=400 \mathrm{GeV}$. We also vary the $A$-terms to obtain a Higgs mass of $125 \mathrm{GeV}$; the few-GeV uncertainty in the Higgs mass translates into an overall factor of $\sim 2$ in the tuning. The UV parameters used to compute tuning for this model are $a_{i}=\left\{M_{3}^{2}, \delta m_{\tilde{t}}^{2}, \delta m_{H_{u}}^{2}, \mu^{2}, A_{t}\right\}$, where $\delta m_{\tilde{t}}^{2}$ and $\delta m_{H_{u}}^{2}$ are the extra contributions to stop and Higgs soft masses, respectively.

The tuning is minimized when the stop mass is close to the gluino mass - lighter stops are more tuned because of the strong RG attractor effect of the gluino (eq. (1.7)). At 
(a) MSSM

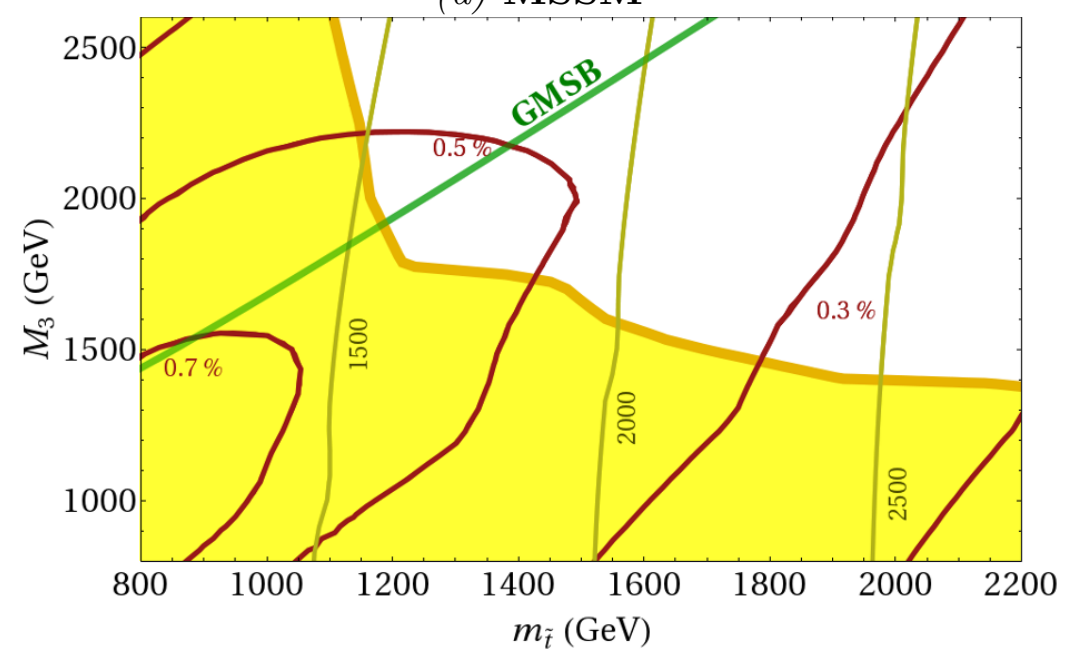

(b) NMSSM

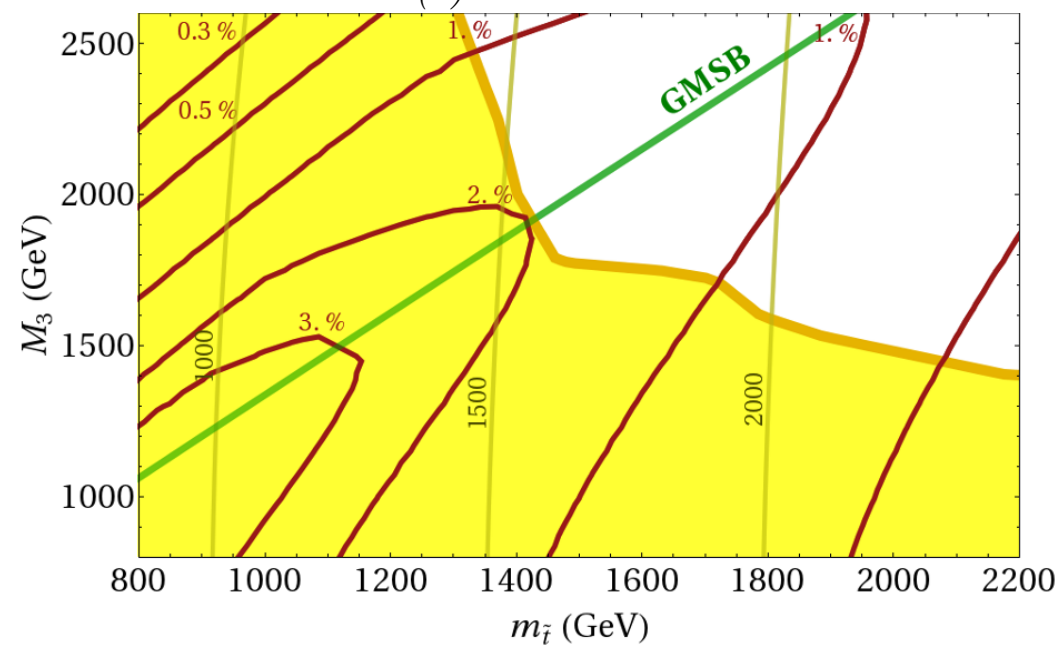

Figure 2. Contours of tuning in the stop-gluino mass plane for the (a) MSSM and the (b) NMSSM models. The vertical golden contours refer to the low energy values of the squark masses generated by universal boundary conditions at the messenger scale $M=300 \mathrm{TeV}$. The green line corresponds to the GMSB boundary conditions for the stop masses with $N=5$ messengers. The $\mu$ term has been fixed to $400 \mathrm{GeV}$. The yellow region corresponds to the direct squark-gluino exclusion bounds from the LHC [14].

a fixed physical stop mass, a smaller gluino mass increases the tuning because a larger $m_{\tilde{t}}^{2}$ at the messenger scale is needed to reproduce the low-energy value. This implies a larger contribution to $m_{H_{u}}^{2}$ for most of the running. These considerations explain the shape of the tuning contours - minimal tuning along the $m_{\tilde{t}} \sim M_{3}$ — which will be qualitatively the same in all of the models studied in the rest of the paper. The plot also shows the current limits on squarks and gluinos from LHC searches. Taking these into account, the tuning amounts to $\sim 0.5 \%$ (up to a factor of 2 in either direction due to uncertainties in the Higgs mass), but is still dominated by the large $A$-terms needed for the Higgs mass. 
To further mitigate the tuning, we need to add another source for the Higgs quartic, as in the NMSSM [17], DMSSM [18], or $\lambda \operatorname{SUSY}[19,20]$. In figure 2(b) we again show contours of fine tuning in the stop-gluino mass plane, taking $a_{i}=\left\{M_{3}^{2}, \delta m_{\tilde{t}}^{2}, \delta m_{H_{u}}^{2}, \mu^{2}\right\}$, now assuming an additional tree-level source for the quartic that fixes the Higgs mass to the measured value in a natural way. ${ }^{3}$ In this case, the LHC bounds on colored sparticles dominate the tuning which is at best $\sim 2 \%$, comparable to that of the MSSM. This is a shift from the pre-LHC era, when the Higgs mass bound from LEP was the primary cause of tuning in supersymmetry.

The LHC thus forces us to move beyond the minimal implementations of SUSY and look for models where the LHC bounds are less stringent. In the following sections, we consider three extensions to the (N)MSSM with GMSB boundary conditions that relax the LHC bounds by increasing the first two generation squark masses (split families), by replacing missing energy with hadronic jets (baryonic RPV), or by reducing collider limits and decoupling the gluino effects in the supersoft limit (Dirac gauginos).

\section{Split families}

\section{$2.1 \quad$ Experimental bounds}

As previously discussed, the most significant contribution to the fine tuning of the EW vacuum comes from the third generation. On the other hand, experimental bounds from direct production at colliders are stronger on the first generation squarks because of the larger production cross section coming from the valence quarks of the proton, while indirect flavor bounds are dominated by $K^{0}-\bar{K}^{0}$ mixing and are significantly stronger on the first two generations. A natural way to relax the tuning is thus to keep the gluino and the third generation squarks light while decoupling the first two generations. These models, introduced well before the LHC [21-23], are known as split-family models.

Given that the first- and second-generation sfermions are heavy, the relevant searches are direct production of gluinos, third-generation sfermions, and electroweak-inos. These channels are well explored by the LHC; the relevant limits are:

- $M_{3} \gtrsim 1.4 \mathrm{TeV}$ for $\tilde{g} \rightarrow t \bar{t} \chi_{1}^{0}$ and $\tilde{g} \rightarrow b \bar{t} \chi_{1}^{+}$, and $M_{3} \gtrsim 1.3 \mathrm{TeV}$ for $\tilde{g} \rightarrow b \bar{b} \chi_{1}^{0}$ [24]. These limits are quite robust over a wide range of split-family spectra, since a light higgsino acts as the LSP in the simplified model. To avoid these bounds, the $\mu$ term has to be larger than $600-700 \mathrm{GeV}$, which leads to $2 \%$ tree-level tuning.

- $m_{\tilde{t}} \gtrsim 700 \mathrm{GeV}$ and $m_{\tilde{b}} \gtrsim 600 \mathrm{GeV}$ [25-27]. However, these limits disappear if the LSP (thus the $\mu$ term) is above $\mu \gtrsim 250-300 \mathrm{GeV}$. Searches for cascade decays into a higgs [28] and those using ISR [29] place limits that are weaker but less dependent on the (N)LSP mass.

\footnotetext{
${ }^{3}$ Explicit implementations of this idea, such as the NMSSM, generically fail to achieve the right value for the Higgs mass in a completely natural way and introduce extra fine-tunings. For this reason our estimate of the tuning from direct bounds should be interpreted as a lower limit.
} 
- Higgsino NLSP: $\mu>330 \mathrm{GeV}$ from higgsino pair production promptly decaying to $Z$ plus MET [30]. The limit might be weakened since $\operatorname{Br}\left(\tilde{h}^{0} \rightarrow Z \tilde{G}\right)$ is model dependent $[31,32]$; however, recent analyses of EWinos decaying to $h$ [33] promise to be almost as sensitive as the those decaying into $Z$. The bound of course disappears if the Higgsino decays outside the detector.

- Stau NLSP: for prompt decays, LEP sets a direct limit of $m_{\tilde{\tau}}>86.6 \mathrm{GeV}$ [34]. Direct limits on promptly decaying staus from the LHC are not as strong, although they can still put very strong indirect bounds (e.g. $M_{3}>1140 \mathrm{GeV}$ [35]). For long-lived staus, the LHC limit is $m_{\tilde{\tau}}>267 \mathrm{GeV}[36,37] .{ }^{4}$

We focus on gauge-mediated models with multiple messengers, which are typically less tuned; therefore we do not consider a bino NLSP, as the stau is a factor of $\sim \sqrt{N_{\text {mess }}}$ lighter than the bino.

\section{$2.2 \quad$ Toy model}

The basic ingredients of split-family models are heavy sfermions of the first two generations and relatively light third-generation sfermions. This can be accomplished in several ways: by using a flavorful $\mathrm{U}(1)[23,38,39]$, localizing the generations on different branes in an extra dimension [40], or different sites in deconstructed versions [41, 42], or by gauging the approximate SU(3) flavor symmetry [43]. Here we are interested in a model that preserves unification and allows for a low mediation scale to minimize the tuning. We achieve this by simply extending the usual gauge mediation mechanism with an additional gauge group, $\mathrm{U}(1)^{\prime}$, under which only the first two generations are charged. By choosing the charge to be $B-L$, the $\mathrm{U}(1)^{\prime}$ is automatically anomaly free, can be implemented at low scales and is compatible with unification. Further details of the model can be found in appendix B.

The resulting sfermion masses consist of a universal gauge-mediated contribution and an additional contribution only to the first two generations:

$$
m_{\phi_{i}}^{2} \sim 2 \sum_{a} C_{a}(i)\left(\frac{\alpha_{a}}{4 \pi} \frac{F}{m_{D}}\right)^{2}+\left(\delta_{1 i}+\delta_{2 i}\right)\left(B_{i}-L_{i}\right)^{2}\left(\frac{\alpha_{\phi}}{4 \pi} \frac{F}{m_{N}}\right)^{2}
$$

where $m_{N}$ and $m_{D}$ are $\mathrm{U}(1)^{\prime}$ and the usual $\mathbf{5}+\overline{\mathbf{5}}$ gauge messenger masses, respectively, and $i$ is the generation index. We absorb the charge of the $\mathrm{U}(1)^{\prime}$ gauge messenger into the definition of the $\mathrm{U}(1)^{\prime}$ gauge coupling $\alpha_{\phi}$. For simplicity we took the same SUSY breaking parameter $F$ for the two contributions, so that the ratio $\alpha_{\phi} m_{D} /\left(\alpha_{a} m_{N}\right)$ controls the mass splitting between the first two and the third generations.

To generate the mixing between the light-flavor and heavy-flavor quarks (e.g. $\mathcal{W} \supset$ $H_{d} Q_{1} D_{3}$ which is forbidden by the $\mathrm{U}(1)^{\prime}$ symmetry), we use another $\mathbf{5}+\overline{\mathbf{5}}$ pair, $D^{\prime \prime}$ and

\footnotetext{
${ }^{4}$ The bound can get as strong as $\sim 400 \mathrm{GeV}$ once other sparticles increase the production cross section [36]. Equivalently, strong indirect bounds can be placed on sparticles such as gluinos, stops, and EWinos that enhance the $\tilde{\tau}$ production cross section.
} 


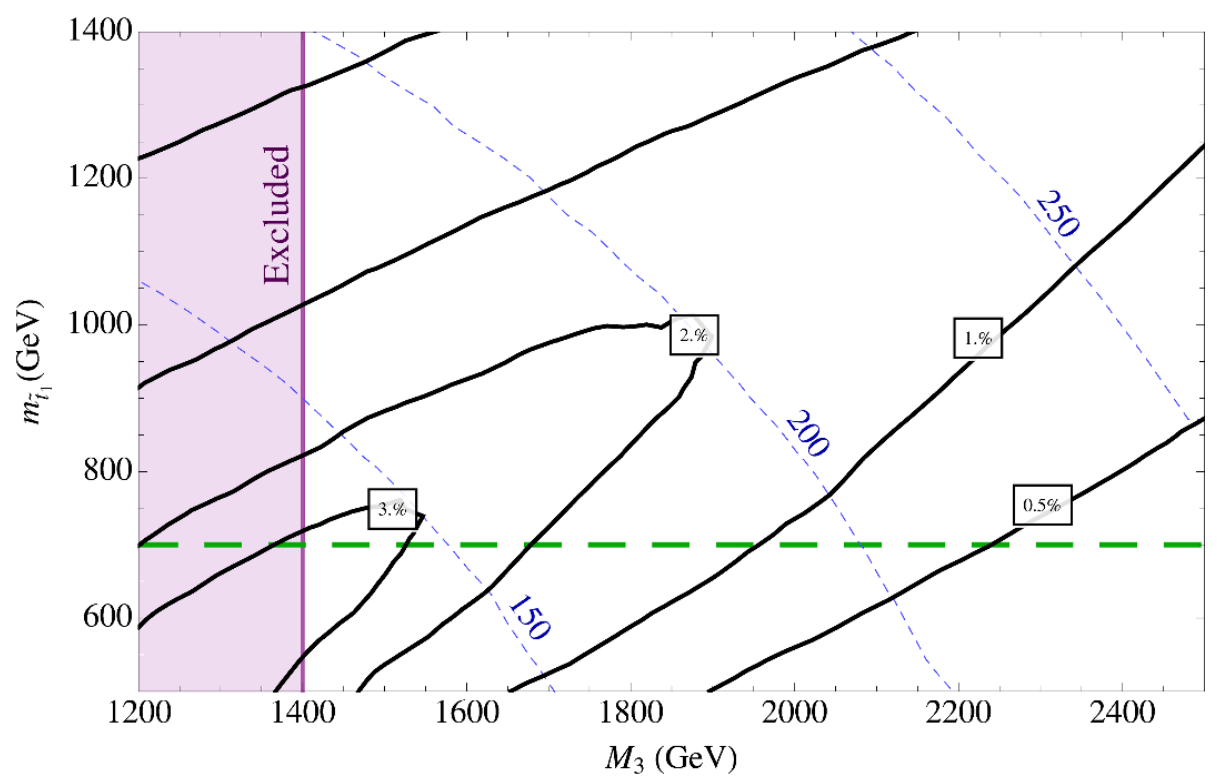

Figure 3. Fine tuning in the split-family model. In this plot, we use 4 pairs of $(5+\overline{5})$ messengers and fix $F / m_{N}^{2}=0.8, \alpha_{\phi}=0.1, \tan \beta=5$, and the first two generations at $\sim 8.5 \mathrm{TeV}$. These parameters determine the mediation scale $m_{D} \sim 10^{7} \mathrm{GeV}$. Additional soft mass contributions to $m_{H_{u}}^{2}$ and $m_{H_{d}}^{2}$ are chosen such that $\mu \sim 300 \mathrm{GeV}$, although the fine tuning is relatively insensitive to the choice of $\mu$ for $\mu \lesssim 400 \mathrm{GeV}$. The solid black lines are FT contours, and the dashed blue lines are the stau mass contours. The shaded region corresponds to the gluino bound, and the dashed green line indicates the limit from direct stop production, which applies for $\mu \lesssim 250 \mathrm{GeV}$.

$\bar{D}^{\prime \prime}$, which does not couple to SUSY breaking, ${ }^{5}$ with superpotential couplings

$$
\mathcal{W} \supset H_{d} Q_{i} D^{\prime \prime}+\Phi \bar{D}^{\prime \prime} D_{3}+m_{D^{\prime \prime}} D^{\prime \prime} \bar{D}^{\prime \prime}+V(\Phi)_{\mathrm{U}(1)^{\prime} \text {-breaking }} \quad(i=1,2) .
$$

$\Phi$ is a singlet under SM gauge groups and breaks the $\mathrm{U}(1)^{\prime}$ symmetry when it acquires a vev. Then the low-energy effective theory below $m_{D^{\prime \prime}}$ contains the necessary $H_{d} Q_{i} D_{3}$ terms.

Split family models generically have non-trivial sflavor structure which can have experimental signatures, e.g. in the $K^{0}-\bar{K}^{0}$ system. In our model, the contributions to flavor observables are within theoretical errors of the SM prediction (see appendix B).

\subsection{Fine tuning}

In figure 3 , we present the fine-tuning with respect to the fundamental parameters: $F$, which characterizes the overall soft mass scale, and $m_{N}$, which characterizes the splitting between the generations. As discussed in section 1.1, we are agnostic to the solution of the $\mu-B \mu$ problem, and therefore additional contributions to $m_{H_{u}}^{2}, m_{H_{d}}^{2}$, and $m_{\tilde{t}}^{2}$ are treated as free parameters and included in the FT calculation.

Split-family models can be less tuned than the (N)MSSM: squark pair production and associated squark-gluino production are effectively turned off if light-flavor squarks

\footnotetext{
${ }^{5}$ When the pair $D^{\prime \prime}$ and $\bar{D}^{\prime \prime}$ is coupled directly to SUSY breaking it generates too large tree-level SUSY breaking contributions.
} 
are decoupled, so the stop can be lighter than the degenerate squark-gluino bound of $\sim 1.6 \mathrm{TeV}$ [14]. However, the improvement is limited because the gluino contribution to fine tuning is not diminished in split-family models. ${ }^{6}$ In particular, the stop mass is still pulled up by the gluino mass; while there is an extra negative contribution to the stop from the heavy first and second generation squarks, using this effect to lower the stop mass is a cancellation between two independent contributions and does not improve the tuning.

We can see this effect when comparing to the fine tuning in the NMSSM in figure 2(b): for a given level of tuning, the maximum gluino mass allowed is comparable between the NMSSM and the split-family model, while the maximum stop mass allowed is typically a few hundred GeV lower in the split families model. Therefore bounds from direct stop pair production, when applicable, are probing the same level of tuning as the gluino bounds.

For $\mu \gtrsim 250 \mathrm{GeV}$, bounds from direct stop pair production no longer apply, but in this case, bounds from pair production of staus provide a complementary probe. Limits on promptly decaying staus are relatively weak, but light staus, especially from cascade decays, are long-lived: the soft masses of the first two generations determine $F \gtrsim\left(\frac{4 \pi}{\alpha_{\phi}} m_{1,2}\right)^{2} \sim$ $10^{12} \mathrm{GeV}^{2}$, which in term determines $c \tau$ of the stau NLSP to be $\gtrsim 1 \mathrm{~m}\left(10^{-3} \mathrm{~m}\right)$ for stau mass of $100 \mathrm{GeV}(300 \mathrm{GeV})$.

While a careful study of the stau bound is beyond the scope of this work, it may actually be the most relevant constraint for the naturalness in this class of models, as can be seen in figure 3. Taking into account all experimental limits, we conclude that split-family models are tuned to at best $3 \%$.

\section{Baryonic RPV}

An experimentally-driven way to reduce limits on sparticles is to eliminate the tell-tale missing energy signature of a stable LSP by allowing some degree of $R$-parity violation (RPV). The presence of both $B$ - and $L$-violating operators would lead to proton decay, and current limits on proton lifetimes set stringent bounds of $10^{-14}-10^{-27}$ on products of $B$ and $L$-violating couplings. In addition, LHC limits on $L$-violating operators are comparable to or stronger than those on the R-parity conserving ones (see e.g. [45, 46]). Given these strong contraints, our best hope of finding natural models in the RPV context lies with models that conserve lepton number and only violate baryon number via the operator $\mathcal{W} \supset \frac{1}{2} \lambda_{i j k}^{\prime \prime} U_{i}^{c} D_{j}^{c} D_{k}^{c}$. There has been a renewed interest in baryonic RPV (bRPV), both in proposed and experimental searches and model-building (see e.g. [47-52] and [53-55]).

\subsection{Constraints on bRPV couplings}

Collider limits. The LEP experiment places limits on charginos $m_{\tilde{\chi}^{ \pm}}>103 \mathrm{GeV}$ independent of the presence of RPV, as well as on stops and sbottoms decaying to jets $m_{\tilde{b}, \tilde{t}} \gtrsim 100 \mathrm{GeV}$ [56]. CMS searches for gluinos decaying to 3 light-flavor jets place limits of $M_{3} \gtrsim 666 \mathrm{GeV}$ with data from the $7 \mathrm{TeV}$ run and a recent ATLAS search for gluinos decaying to decaying to $t \tilde{t}^{*}$ with $\tilde{t}^{*} \rightarrow b s$ sets a gluino limit of $860 \mathrm{GeV}$ for stop masses up

\footnotetext{
${ }^{6}$ For another analysis of tuning in split families, see [44].
} 
to $1 \mathrm{TeV}[51,52,57]$. Even more recently [58], ATLAS searches for pair-produced gluinos decaying to 6 or 10 jets set limits between $\sim 650 \mathrm{GeV}$ and $\sim 950 \mathrm{GeV}$ depending on the final state quarks; including the associated squark-gluino production in the cross-section could raise the limits to over a $\mathrm{TeV}$. While these searches place important constraints, they are preliminary and sensitive to the sparticle spectrum and value of $\lambda_{i j k}^{\prime \prime}$ couplings. Other searches which are used for R-parity conserving SUSY can be recast to place limits on hadronic RPV spectra; for instance multijet and same-sign lepton searches were used in [59] to set limits of $800-900 \mathrm{GeV}$ on gluinos and first- and second-generation squarks with just the $7 \mathrm{TeV}$ dataset.

Low-energy limits. While LHC experiments are only recently beginning to search for RPV decays of sparticles, there is a multitude of indirect limits on $B$-violating couplings from rare decays and low-energy experiments (for a review see [45]):

- The missing energy signature at colliders can be restored if the LSP decays out of the detector, or the NLSP has a sizable decay width to a light gravitino. The former requires, for neutralino 3-body decays,

$$
\lambda_{i j k}^{\prime \prime}>7 \times 10^{-6}\left(\frac{m_{\tilde{\chi} 0}}{125 \mathrm{GeV}}\right)^{-5 / 2}\left(\frac{m_{\tilde{q}}}{600 \mathrm{GeV}}\right)^{2}\left(\frac{1 \mathrm{~m}}{c \tau}\right)^{1 / 2}\left(\frac{\zeta}{0.1}\right)^{-1}
$$

where $\zeta$ is the neutralino-squark-quark coupling. Two-body right-handed squark decays give

$$
\lambda_{i j k}^{\prime \prime}>10^{-9}\left(\frac{m_{\tilde{q}}}{600 \mathrm{GeV}}\right)^{-1}\left(\frac{1 \mathrm{~m}}{c \tau}\right)^{1 / 2},
$$

while left-handed squark decays are further suppressed by left-right mixing; we will focus on a neutralino NSLP because of naturalness. We discuss limits from decay into the gravitino in the following section.

- Violation of baryon number washes out the results of baryogenesis unless $\lambda_{i j k}^{\prime \prime}<10^{-7}$; if the reheat temperature is low these bounds can be avoided [60, 61]. Recently, it has been pointed out that the baryogenesis problem in RPV models can be also addressed without invoking a low reheat temperature [62].

- Low-energy measurements such as neutron-antineutron oscillations and di-nucleon decay; the relevant limit for our discussion is $\lambda_{1 j k}^{\prime \prime} \lesssim 10^{-5}\left(\frac{m_{\tilde{q}}}{600 \mathrm{GeV}}\right)^{2}\left(\frac{M_{3}}{600 \mathrm{GeV}}\right)$ [63].

Some couplings, e.g. $\lambda_{323}^{\prime \prime}$, have no direct experimental constraints; however, a concrete model of RPV couplings connects these to more constrained couplings. In order to avoid flavor constraints, generic models will relate the UDD R-parity violating couplings to powers of the corresponding yukawa couplings. In the model we consider below, for example, the couplings that provide the strongest constraints are the first generation of up-type couplings $\lambda_{1 j k}^{\prime \prime}$. The relevant limits are shown in figure 4 , and more details can be found in appendix $\mathrm{C}$. 


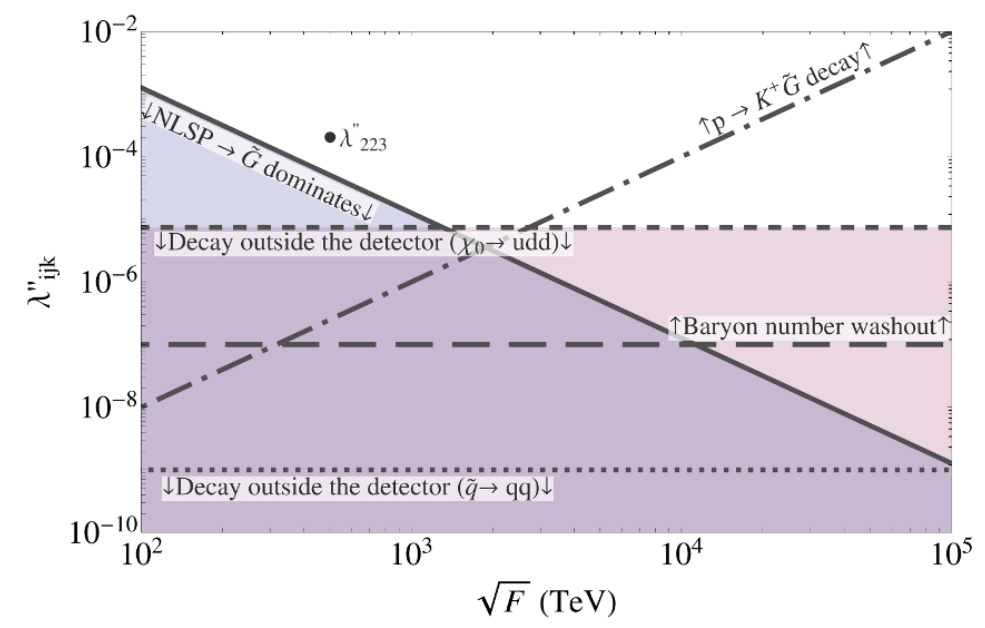

Figure 4. Limits on RPV UDD coupling $\lambda_{i j k}^{\prime \prime}$ and scale of SUSY breaking $\sqrt{F}$ when one coupling $\lambda_{\max }^{\prime \prime}$ dominates. The region below the dashed line is excluded as the NLSP would decay outside the detector, restoring the MET signature (if the only available decay is 3-body). For low $\sqrt{F}$, the decay rate to the gravitino can exceed the RPV decay rate (solid line), again leading to MET. Baryogenesis and proton decay bounds are indicated by the thick-dashed and the dot-dashed lines, respectively. There may be up to $\mathcal{O}(10)$ uncertainty in some of the bounds presented. Without extra assumptions, the entire parameter space is excluded; we assume low-scale baryogenesis and an additional SUSY-breaking sector to raise the gravitino mass and avoid proton decay. Since the NLSP is below the top threshold, the LHC phenomenology of the toy model is controlled by the 2nd generation coupling $\lambda_{2 j k}^{\prime \prime}$, and we use a low SUSY-breaking scale of $\sqrt{F}=500 \mathrm{TeV}$, as indicated by the gray point.

The gravitino. In theories with low-scale SUSY breaking (as favored by naturalness), there is a light gravitino with mass $m_{\tilde{G}} \gtrsim \mathrm{keV} \times \frac{F}{\left(10^{3} \mathrm{TeV}\right)^{2}}$. In the presence of RPV, proton decay via $p \rightarrow K^{+} \tilde{G}$ results in a strong constraint on UDD couplings, which is particularly stringent on light-flavor RPV:

$$
\lambda_{i j k}^{\prime \prime} \lesssim 10^{-6} \frac{F}{\left(10^{3} \mathrm{TeV}\right)^{2}}\left(\frac{m_{\tilde{s}_{R}}}{600 \mathrm{GeV}}\right)^{3}, \quad \text { and } \quad \lambda_{112}^{\prime \prime} \lesssim 10^{-12} \frac{F}{\left(10^{3} \mathrm{TeV}\right)^{2}}\left(\frac{m_{\tilde{s}_{R}}}{600 \mathrm{GeV}}\right)^{2}
$$

With all RPV couplings less than $\mathcal{O}\left(10^{-6}\right)$, sparticle RPV decays compete with decays to the light gravitino. In case the gravitino decay is order one - or even $10 \%$ - the missing energy signature of $R$-parity conserving SUSY is effectively restored. ${ }^{7}$ This constraint restricts the scale of SUSY breaking through the decay rate to the gravitino,

$$
\lambda_{\max }^{\prime \prime} \gtrsim 4 \times 10^{-5}\left(\frac{F}{10^{6} \mathrm{TeV}^{2}}\right)^{-1}\left(\frac{m_{\tilde{q}}}{600 \mathrm{GeV}}\right)^{2}\left(\frac{\zeta}{0.1}\right)^{-1}\left(\frac{\operatorname{Br}\left(\tilde{\chi}_{0} \rightarrow \tilde{G} X\right)}{10 \%}\right)^{-1 / 2} .
$$

where $\lambda_{\max }^{\prime \prime}$ is the coupling that dominates the neutralino RPV decay. Given the strong constraints from equations (3.3) and (3.4), it is useful to consider a gravitino mass above the

\footnotetext{
${ }^{7}$ The relevant searches have efficiencies that are relatively insensitive to the superpartner masses (e.g. photon and missing energy [64]), so the scale of SUSY breaking must be such that the branching ratio to gravitino times the production cross-section is less than the experimental limit on $\sigma$ (a few fb).
} 
proton mass. A gravitino heavier than $1 \mathrm{GeV}$ can decay through baryon number violation to three quarks which then hadronize, $\tilde{G} \rightarrow u_{i} d_{j} d_{k}$, with a lifetime

$$
\tau_{\tilde{G}} \simeq 10^{7} \mathrm{~s}\left(\frac{10 \mathrm{GeV}}{m_{\tilde{G}}}\right)^{9}\left(\frac{m_{\tilde{q}}}{600 \mathrm{GeV}}\right)^{4}\left(\frac{\sqrt{F}}{100 \mathrm{TeV}}\right)^{4}\left(\frac{10^{-4}}{\lambda_{2 j k}^{\prime \prime}}\right)^{2} .
$$

Note that here $F$ of our sector can be less than $m_{\tilde{G}} M_{\mathrm{pl}}$, so the decay is relatively faster. The lifetime ranges from as short as $10^{-5} \mathrm{~s}$ for $\lambda_{2 j k}^{\prime \prime}=0.1$ and a $20 \mathrm{GeV}$ gravitino, to longer than $10^{19}$ s for a $2 \mathrm{GeV}$ gravitino with $1200 \mathrm{GeV}$ squarks and $F$ of $\left(10^{3} \mathrm{TeV}\right)^{2}$.

Even without committing to a particular hierarchy of RPV couplings, it is impossible to satisfy all the constraints discussed above (as shown in figure 4). We choose to give up the limit from baryogenesis. We also introduce extra hidden sector(s) to avoid proton decay limits while keeping a low mediation scale; these sectors have SUSY breaking which lifts the gravitino mass up to $1-15 \mathrm{GeV}$ while the $F$ corresponding to the SUSY breaking scale in our sector is lower. To avoid the limits discussed above, we need the gravitino with $m_{\tilde{G}}>1 \mathrm{GeV}$, but not too heavy: flavor problems from gravity mediation arise for $m_{\tilde{G}} / m_{\tilde{q}} \gtrsim$ $0.02 \times \sqrt{m_{\tilde{q}} / \mathrm{TeV}}$, while for higher gravitino mass, the gravity-mediated contributions dominate the tuning due to the very large amount of running.

\section{$3.2 \quad$ Toy model}

Given the constraints discussed above, we impose the following requirements on a model of R-parity violation: gauge coupling unification; no lepton-number violation; low scale of SUSY breaking to reduce fine-tuning; gauge mediation to avoid flavor issues; and a hierarchy of couplings between the very constrained first-generation $\lambda_{1 j k}^{\prime \prime}$ couplings and the less constrained $\lambda_{2 j k}^{\prime \prime}, \lambda_{3 j k}^{\prime \prime}$ couplings.

We use an orbifold GUT model with $\mathrm{SU}(5)$ and matter in the bulk, and $\mathrm{SU}(3) \times$ $\mathrm{SU}(2) \times \mathrm{U}(1)$, the Higgses, and B-violation on the IR brane. Doublet-triplet splitting solutions in these models allow us to achieve baryon breaking number without lepton number while preserving the prediction of unification. This results in a predictive pattern of RPV couplings,

$$
\lambda_{i j k}^{\prime \prime} \simeq \frac{\langle\Phi\rangle^{2}}{M_{D}^{2}} \sqrt{y_{i}^{u}}\left(\frac{y_{j}^{d}}{\sqrt{y_{j}^{u}}}\right)\left(\frac{y_{k}^{d}}{\sqrt{y_{k}^{u}}}\right),
$$

where $M_{D}$ is the mass of a heavy $D$-type field and the vev of $\Phi$ breaks baryon number. For more details of the model, see appendix C.

The pattern of RPV couplings results in the limits of

$$
\lambda_{1 j k}^{\prime \prime} \lesssim 10^{-5}, \quad \lambda_{2 j k}^{\prime \prime} \lesssim 2 \times 10^{-4}, \quad \text { and } \quad \lambda_{3 j k}^{\prime \prime} \lesssim 3 \times 10^{-3}
$$

for squark and gluino masses of $600 \mathrm{GeV}$ or higher. In order to minimize the tuning, we maximize the value of $\langle\Phi\rangle^{2} / M_{D}^{2}$ — thus the RPV couplings - to minimize the branching ratio of the NLSP to the goldstino and achieve the lowest SUSY breaking scale possible. 


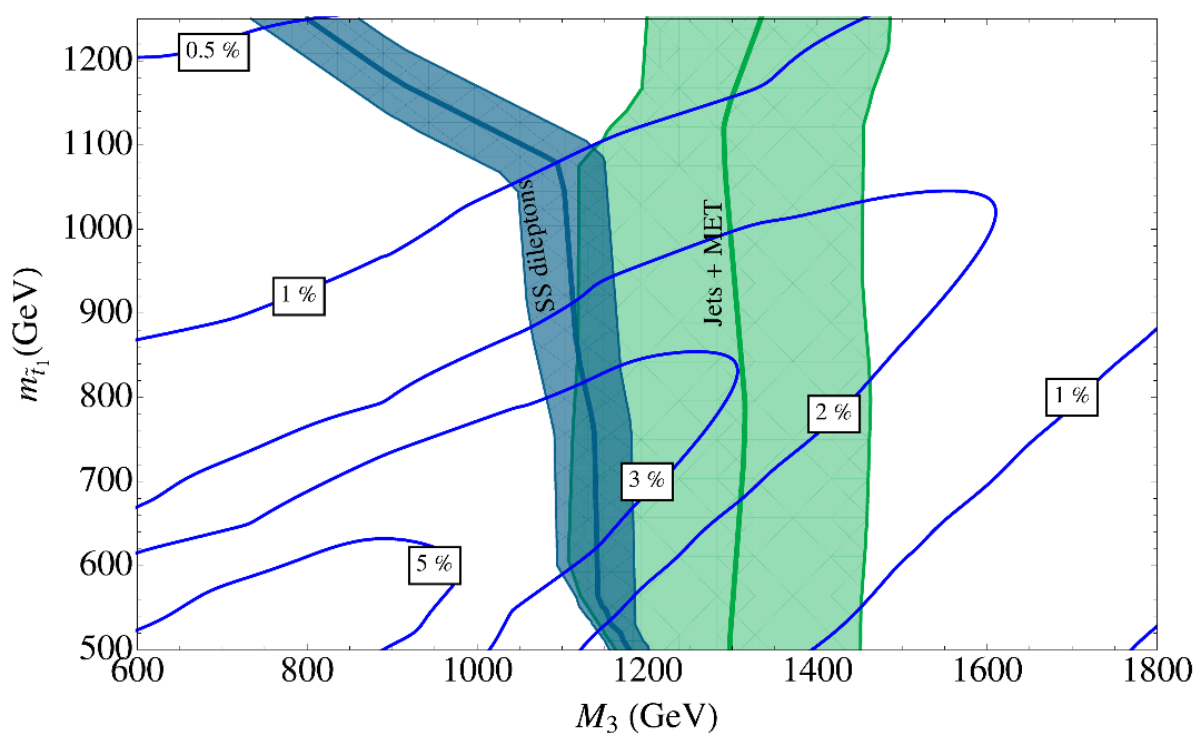

Figure 5. Tuning in RPV with standard gauge-mediated gaugino masses and $\mu=125 \mathrm{GeV}$. The blue band is the recast CMS same-sign dilepton limit $\left(10 \mathrm{fb}^{-1}\right)$ [68], and the green band is the recast ATLAS 7-10 jets and MET limit $\left(20 \mathrm{fb}^{-1}\right)$ [65]. The width of the band is the uncertainty in our estimate; see appendix $\mathrm{C}$ for details. The NLSP is a neutralino which decays through bRPV, $\chi_{0} \rightarrow U D D$.

\subsection{Fine tuning}

The most stringent limits come from multiple jet and MET searches [65, 66] as well as leptonic searches $[57,67]$. These are shown in figure 5; for details of event simulations, analysis, and validation, as well as comparison to other re-castings, see appendix C. We calculate fine tuning against UV parameters as follows,

$$
\mathrm{FT}_{\text {total }}=\mathrm{FT}_{m_{\tilde{t}}^{2}}\left[\delta m_{H_{u}}^{2}, \delta m_{\tilde{t}}^{2}, \Lambda^{2}\right] \times \mathrm{FT}_{m_{h}^{2}}\left[\mu, \delta m_{H_{u}}^{2}, \delta m_{\tilde{t}}^{2}, \Lambda^{2}\right]
$$

In generic spectra, leptons and missing energy resulting from electroweakino and top decays are relatively common in cascades. The NLSP is a combination of bino and higgsino and decays predominantly through $\lambda_{2 j k}^{\prime \prime}$ to three jets, possibly including a $b$-jet. We fix the $\mu$ term at $125 \mathrm{GeV}$, and the benefit is two-fold: light higgsinos are beneficial for naturalness, and an NLSP below the top mass reduces the number of leptons in cascades, as otherwise the NLSP would decay dominantly to a top through the larger third-generation $\lambda_{3 j k}^{\prime \prime}$ couplings. The limits on the gluino and squark masses are much reduced compared to R-parity-conserving scenaria; however, the tuning of the models is still at the few percent level as shown in figure 5 .

There are two avenues to try to further reduce the bounds. One is to remove leptons and neutrinos completely from the decay chain, but this generally leads to increased tuning. For example, we found that a spectrum with a heavy wino has comparable limits from same-sign (SS) leptons to figure 5, but tuning increases by a factor of 2 to 3 due to the larger left-handed stop mass and the addition of an extra parameter. The leptons in this 
case arise from e.g. $\tilde{g} \rightarrow \tilde{b}_{R} b \rightarrow \tilde{\chi}^{-} t b$ decays as well as transitions between the remaining electroweakinos through $W \mathrm{~s}$ and $Z \mathrm{~s}$. The latter transitions can come from off-shell decays of the charged higgsinos to the neutral higgsino, which still dominate over direct RPV decays due to a relatively small RPV coupling. Another possibility is a very light spectrum where decays through on-shell tops are kinematically forbidden, for example higgsinos at $150 \mathrm{GeV}$, squarks at $300 \mathrm{GeV}$ and gluinos at $450 \mathrm{GeV}$. However, this scenario is ruled out by ATLAS multijet searches for RPV gluinos $[52,58] .{ }^{8}$

Another approach is to reduce the very large colored production cross-sections by decoupling the first two generation squarks as in split families. However, a light gluino is still required by naturalness; such a split RPV spectrum is covered by an ATLAS search for $\tilde{g} \rightarrow \tilde{t} \bar{t}$, with $\tilde{t} \rightarrow j j$ as mentioned previously [57], resulting in a limit of $860 \mathrm{GeV}$ on the gluino mass from SS leptons, assuming the only leptons arise from a top on either side of the decay chain. Introducing new sources of leptons in the cascade will increase the bound. In particular, a light higgsino, required for naturalness, would further increase this limit by adding more tops to the cascade: the stops will decay $\tilde{t} \rightarrow \tilde{H}^{0} t$ with a large rate due to the large yukawa coupling, increasing the incidence of SS leptons by nearly a factor of 4 which would raise the limit to $1050 \mathrm{GeV}$ for gluino production alone. In addition, by assumption the bino and wino are decoupled, which further increases the tuning, and the mechanism to split the families also introduces more tuning cf. section 2.

One alternative we have not considered so far is a stop NLSP that decays to two jets via a UDD coupling. Here we can apply the bound of [57] at face value, so a $860 \mathrm{GeV}$ gluino is allowed; the optimal tuning in our model would be about $5 \%$ for a $600 \mathrm{GeV}$ stop. However, this does not take into account the remainder of the tunings required to achieve this spectrum. First, the cross-section used to set the above limit assumes only gluino pairproduction; splitting the families to tends to further increase the tuning, or alternatively, the increased production cross-section from the presence of light squarks leads to a limit of $1100 \mathrm{GeV}$ on the average gluino-squark mass. Also, as discussed above, it is necessary to raise the higgsinos to avoid additional tops in the cascade. Fixing the $\mu$ term at $400 \mathrm{GeV}$ or higher to reduce the branching fraction of $\tilde{t} \rightarrow \tilde{H}^{0} t$ gives $5 \%$ tuning from the $\mu$ term; alternatively, lowering the stop mass against the gluino mass introduces additional tuning of at least a factor of $\left(600 / m_{\tilde{t}}\right)^{2}$. These values do not take into account adding these tunings in quadrature with other sources, such as new parameters to decouple the wino and bino or the sleptons. In fact, including only the tunings due to the $\mu$ term and to splitting the families, a stop at $600 \mathrm{GeV}$ and higgsino at $400 \mathrm{GeV}$ result in $\mathrm{FT} \sim 3.5 \%$, at the same level as the neutralino NLSP case we discuss above.

\section{Dirac gauginos}

One possible way to extend the MSSM is to impose an exact $R$-symmetry, and - as Majorana gaugino masses are now forbidden - to postulate that the gauginos $\lambda_{i}$ acquire Dirac mass terms with the fermions of extra adjoint superfields $A_{i}$ [69-75]. $R$-symmetric Dirac gaugino models have been heralded as beneficial for fine-tuning of the Higgs mass [72,

\footnotetext{
${ }^{8}$ We thank Prashant Saraswat for confirming that such light spectra are excluded.
} 
76]. The stops still contribute to the Higgs mass and quartic, but the gluino can be naturally heavy without "pulling up" the squark masses, removing the dominant contribution to finetuning given the strong LHC bound on the gluino mass [16]. Bounds on direct squark pair production are milder, because $t$-channel gluino exchange decouples faster for a heavy Dirac gluino than for a heavy Majorana gluino [77]. Finally, flavor bounds are significantly weakened in the absence of Majorana gaugino masses [78].

From the outset unification is difficult to achieve, as the $\mathrm{SU}(2)$ and $\mathrm{SU}(3)$ adjoint fields dramatically change the running of the gauge couplings (see [79] for a one-loop analysis). In particular, at scales above the mass of the chiral octet $A_{3}$ the strong gauge coupling is no longer asymptotically free, and at two-loop order runs stronger according to:

$$
\partial_{t} \alpha_{3}=0 \alpha_{3}^{2}+\frac{136}{(4 \pi)^{2}} \alpha_{3}^{3}+\ldots
$$

To prevent a Landau pole before the scale $10^{16} \mathrm{GeV}$ (which would lead to unacceptably rapid proton decay) any pair of colored states needs to be heavier than $\sim 10^{12} \mathrm{GeV}$. The minimal representation of $\mathrm{SU}(5)$ containing the $A_{i}$ is the $\mathbf{2 4}$, which also has "bachelor" fields $(3,2,-5 / 6)+(\overline{3}, 2,5 / 6)$. The mass of these bachelor fields has to be above $\sim 10^{14} \mathrm{GeV}$ to avoid a Landau pole before $10^{16} \mathrm{GeV}$. It is also possible to achieve perturbative unification with additional light electroweak states in incomplete multiplets. In either case, the quantitative prediction of gauge coupling unification in the MSSM is lost, since additional states need to be added at intermediate scales.

The above-mentioned positive aspects of Dirac gaugino models stem from the "supersoft" operator

$$
\mathcal{W} \supset \sqrt{2} m_{D i} \theta^{\alpha} W_{i \alpha}^{a} A_{i}^{a} \quad \Rightarrow \quad \mathcal{L} \supset-m_{D i} \lambda_{i}^{a} \tilde{A}_{i}^{a}-\sqrt{2} m_{D i}\left(A_{i}^{a}+A_{i}^{a \dagger}\right) D_{i},
$$

which contains Dirac masses for the gauginos labeled by $i=1,2,3$. After integrating out the auxiliary $D$-terms, it also gives rise to mass terms for the real component of the scalar adjoints, $\operatorname{Re}\left(A_{i}^{a}\right)$, and a tri-scalar coupling of $\operatorname{Re}\left(A_{i}^{a}\right)$ with the MSSM sfermions. In addition, the scalar adjoints can also have SUSY-breaking, $R$-symmetry-preserving mass terms:

$$
\mathcal{L} \supset m_{A_{i}}^{2} A_{i}^{a \dagger} A_{i}^{a}+B_{A_{i}}\left(A_{i}^{a} A_{i}^{a}+\text { h.c. }\right) .
$$

From eqs. (4.2) and (4.3), the real and imaginary components of the adjoint scalars have masses

$$
m_{\operatorname{Re}\left(A_{i}\right)}^{2}=4 m_{D i}^{2}+m_{A_{i}}^{2}+B_{A_{i}} \quad m_{\operatorname{Im}\left(A_{i}\right)}^{2}=m_{A_{i}}^{2}-B_{A_{i}} .
$$

The sfermion masses do not receive any log-divergent one-loop corrections proportional to $m_{D i}$, but do get finite threshold contributions due to the mass splittings of the Dirac gauginos and their real scalar adjoints [72]:

$$
\Delta_{\text {finite }} m_{\tilde{f}}^{2}=\sum_{i} \frac{C_{i}(f) \alpha_{i} m_{D i}^{2}}{\pi} \log \frac{m_{\operatorname{Re}\left(A_{i}\right)}^{2}}{m_{D i}^{2}} .
$$


LHC bounds on Dirac gaugino models can be relatively mild if Dirac gluino pair production and associated production are not kinematically accessible. The cross-section for direct squark pair production is reduced due to the suppression of the $t$-channel (Dirac) gluino exchange diagram, leading to reduced limits for colored sparticles at the LHC [76, 77]. In a squark-LSP simplified model with decoupled gluinos, CMS and ATLAS place bounds of $m_{\tilde{q}} \gtrsim 800 \mathrm{GeV}$ if $m_{\mathrm{LSP}} \lesssim 300 \mathrm{GeV}$ with $\sim 20 \mathrm{fb}^{-1}$ of data [14,80]; CMS has an earlier search using razor variables which excludes $m_{\tilde{q}} \lesssim 600 \mathrm{GeV}$ for heavier LSP masses as well [81]. Very compressed spectra with low masses may still be allowed, but since they are not generic in Dirac gaugino models we do not consider these here. The NLSP is typically the higgsino or a right-handed slepton; the gravitino may also be relevant for collider limits depending on the scale of SUSY breaking. If the NLSP is a higgsino and decays to a gravitino via a $Z$ boson inside the detector, the higgsino mass is constrained to be $\mu \gtrsim 360 \mathrm{GeV}$ [82]. Direct searches for sleptons [30] can be important as well depending on their masses and the decay width to the gravitino, although they are less relevant for naturalness of Dirac gaugino models.

In gauge-mediated models of Dirac gauginos [72, 83-85], it is assumed that the UV soft mass terms in eqs. (4.2) and (4.3) arise from a perturbative coupling of the adjoint superfields with sets of messengers $X_{j}, X_{j}^{c}$ in the superpotential $\mathcal{W} \supset M_{j k} X_{j} X_{k}+$ $\sum_{i} y_{i j k} X_{j}^{c} A_{i} X_{k}$. If the messengers are charged under a $\mathrm{U}(1)^{\prime}$ that acquires a $D$-term expectation value, $m_{D_{i}}, m_{A_{i}}^{2}$, and $B_{A_{i}}$ are generated at one loop [83, 84]. In a minimal model with one pair of messengers, $m_{A_{i}}^{2}$ is suppressed at leading order in $D^{2} / M^{2}$, which causes the real scalar adjoint to be tachyonic. However, it is possible to give positive masses to both the real and imaginary scalar adjoints with multiple sets of messengers provided there is sufficient mixing between them [83], or with additional ( $R$-symmetric) $F$-terms [79]. Mediation schemes with both non-tachyonic adjoints thus naturally generate the mass terms $m_{A_{i}}^{2}$ and $B_{A_{i}}$ a loop factor higher than $m_{D i}^{2}$.

These models of Dirac gauginos are not supersoft at higher orders. In particular, if $m_{A_{i}}^{2} \neq 0$, there is a large two-loop contribution to the squark masses of the form $[86,87]:^{9}$

$$
\partial_{t} m_{\tilde{q}}^{2} \simeq \frac{32 \alpha_{3}^{2}}{(4 \pi)^{2}} m_{A_{3}}^{2}+\ldots
$$

This RG effect drives the squarks tachyonic and can dominate the positive, finite contribution in eq. (4.5). This means that the ratio $m_{A_{3}}^{2} / m_{D 3}^{2}$ at the SUSY-breaking scale $\Lambda$ needs to be sufficiently small to avoid tachyonic or unacceptably light squarks. ${ }^{10}$ The generic gauge mediated prediction of this ratio - an inverse loop factor - results in tachyons for low and intermediate mediation scales; we show squark mass contours as a function of $\Lambda$ and $m_{A_{3}}^{2} / m_{D 3}^{2}$ in figure 6 . We also show mass contours of the right-handed sleptons, under the assumption of degenerate adjoint mass terms and $m_{D i} / m_{D j}=g_{i} / g_{j}$ at the scale $\Lambda$.

\footnotetext{
${ }^{9}$ The first version of [86] differed from eq. (4.6) by a factor of $8 / 3$.

${ }^{10}$ Electroweak symmetry breaking may also place a bound on the ratio $m_{A_{3}}^{2} / m_{D 3}^{2}$ in explicit models, because above the approximate threshold scale $\sqrt{m_{D 3} m_{A_{3}}}$, the stop mass is negative when $m_{A_{3}}^{2}>0$, giving positive contributions to $m_{H_{u}}^{2}$.
} 


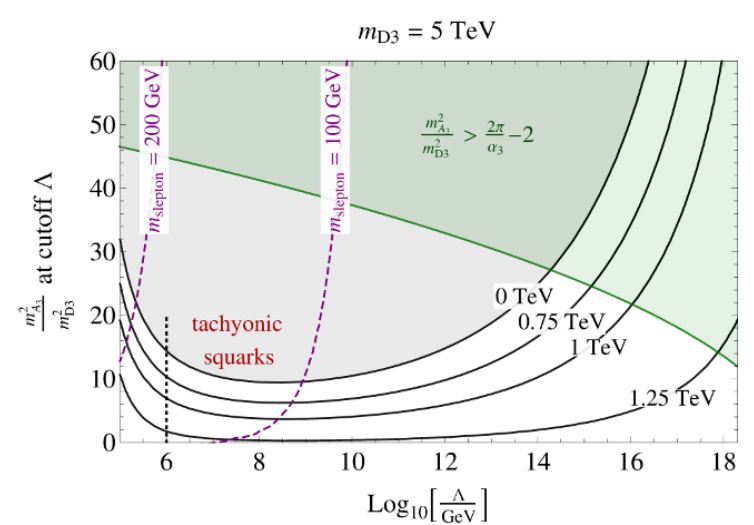

(a)

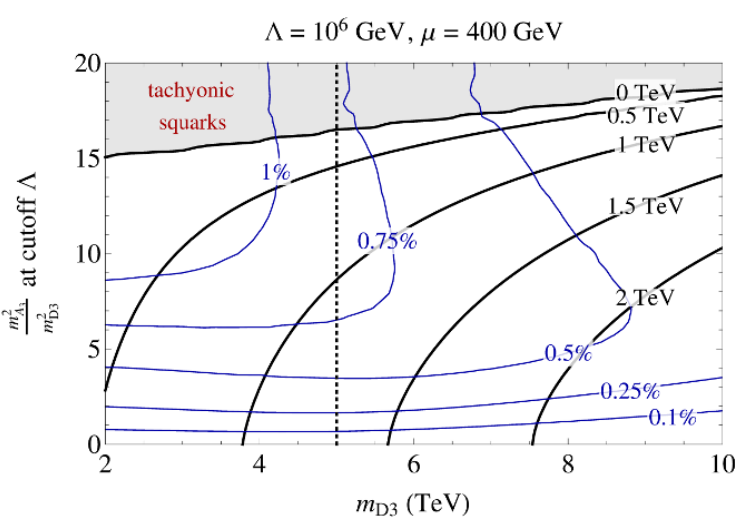

(b)

Figure 6. (a) Squark mass contours (black) as a function of the mediation scale $\Lambda$ and the ratio $m_{A_{3}}^{2} / m_{D 3}^{2}$ at the scale $\Lambda$, with the physical Dirac gluino mass fixed at $5 \mathrm{TeV}$ and adjoint scalar masses as in eq. (4.4) with $B_{A_{i}} \approx 0$. Contours of right-handed slepton masses are shown in purple under assumptions given in the text. The region $m_{A_{3}}^{2} / m_{D 3}^{2}>\frac{2 \pi}{\alpha_{3}}-2$ as in the pure $D$-term model of [83] is shown in green; note that its contour changes with $\Lambda$ due to eq. (4.1). (b) Squark mass (black) and fine tuning (blue) contours as a function of the physical Dirac gluino mass $m_{D 3}$ and the ratio $m_{A_{3}}^{2} / m_{D 3}^{2}$ at the mediation scale $\Lambda=10^{6} \mathrm{GeV}$. The black dotted line on each figure represent 1D slices of the other figure.

It may be possible to construct a low-scale model in which $m_{D i}^{2} \sim m_{A_{i}}^{2} \sim B_{A_{i}}$ with the correct relative signs; however, we expect such an arrangement to be accompanied with additional fine tuning given the natural loop suppression of $m_{D i}^{2}$ vs. $m_{A_{i}}^{2}$. We quantify the total fine tuning in such a low-scale model in figure $6(\mathrm{~b})$, where we assumed:

$$
\mathrm{FT}_{\text {total }}=\frac{m_{A_{3}}^{2} / m_{D 3}^{2}}{\pi / \alpha_{3}} \times \mathrm{FT}_{m_{h}^{2}}\left[a_{i}\right],
$$

with $a_{i}=\left\{\mu^{2}, m_{H_{u}}^{2}, m_{D 3}^{2}, m_{A_{3}}^{2}\right\}$. We take $\mu=400 \mathrm{GeV}$ in figure $6(\mathrm{~b})$ to satisfy the bounds on higgsinos in models of low-scale gauge mediation. We find that low-scale models of Dirac gauginos are tuned to at least $1 \% .^{11}$

For sufficiently high mediation scales, $\Lambda \gtrsim 10^{15} \mathrm{GeV}$, the squark tachyon problem can be avoided more easily. This is because $m_{A_{3}}^{2}$ is irrelevant in the IR compared to $m_{D 3}^{2}$ and $B_{A_{3}}$; at one-loop order with preserved $R$-symmetry, the $\beta$-functions are:

$$
\partial_{t} m_{A_{3}}^{2}=0, \quad \frac{\partial_{t} m_{D 3}^{2}}{m_{D 3}^{2}}=\frac{\partial_{t} B_{A_{3}}}{B_{A_{3}}}=-\frac{3 \alpha}{\pi} .
$$

These relations imply that the theory flows to the supersoft limit $\left(\left|m_{D 3}^{2}\right|,\left|B_{A_{3}}\right| \gg\left|m_{A_{3}}^{2}\right|\right)$ in the IR, with only moderate contributions from $m_{A_{3}}^{2}$ to the squark masses (eq. (4.6)). To avoid tachyonic octet scalars at low scales, it is sufficient that $4 m_{D 3}^{2}>-B_{A_{3}}>0$ at the mediation scale (eqs. (4.4) and (4.8)). However, RG flow from e.g. $\Lambda \sim 10^{16} \mathrm{GeV}$ with

\footnotetext{
${ }^{11}$ If one considers only the observable $m_{h}^{2}$ and not $m_{A_{3}}^{2} / m_{D 3}^{2}$, the tuning is at best $3.5 \%$, e.g. for the parameters $m_{D 3}=4 \mathrm{TeV}, m_{A_{3}}^{2} / m_{D 3}^{2} \approx 0, \mu=400 \mathrm{GeV}$, and $\Lambda=10^{6} \mathrm{GeV}$.
} 
universal gaugino mass boundary conditions creates a factor of $\gtrsim 15$ splitting between the gluino and bino masses, yielding $\gtrsim 5 \mathrm{TeV}$ squark masses if right-handed sleptons are above $\sim 100 \mathrm{GeV}$. A combination of $F$ - and $D$-terms can give $\mathcal{O}(100 \mathrm{GeV})$ masses to the sleptons and $\mathcal{O}(1 \mathrm{TeV})$ masses to the squarks at low scales. However, a typical feature of highscale mediation models is that the octet superfield $A_{3}$ acquires an $R$-symmetry-breaking superpotential mass $M_{A_{3}}$ via gravity mediation.

This mass term is by far the most relevant operator in the IR: $\partial_{t} M_{A_{3}} / M_{A_{3}} \simeq-6 \alpha_{3} / \pi$. When dominant, it yields a light gluino mass eigenstate of mass $\sim m_{D 3}^{2} / M_{A_{3}}$ at low scales through a seesaw mechanism. Hence the natural separation between the stop and Dirac gluino mass is destroyed, so naturalness is again spoiled due to the strong LHC bounds on the gluino.

We did not touch upon other model-building challenges — such as avoiding a vev for the $\mathrm{SU}(2)$ adjoint scalar and generating large enough effective $\mu, B \mu$, and Higgs quartic coupling — which have been covered extensively in bottom-up studies [85, 88]. In particular, one additional contribution to the tuning of the Higgs mass could come from operators such as $\mathcal{W} \supset H_{u} A_{1,2} H_{d}$ especially if $A_{1}$ and $A_{2}$ are heavy [86]. These obstacles notwithstanding, it is clear from figure 6 and the above discussion that the colored sector controlled by the Dirac gluino and colored octet mass parameters - must be very peculiar to obtain a viable, let alone natural, model of Dirac gauginos.

\section{Summary}

The previous sections show that naturalness of the EW scale is being severely challenged by the LHC. Even if the Higgs sector is extended to explain the measured value of $125 \mathrm{GeV}$, as in the NMSSM, the tuning imposed by direct searches of colored sparticles is at the percent level, not much improved compared to the ordinary MSSM with large A-terms (see table 1). To have a chance of improving the situation we need to go beyond the minimal model and look for mechanisms that explain the absence of signals at the LHC. Unfortunately, all the alternatives proposed so far in the literature seem to fail mainly because of the strong bounds on the gluino mass.

Because of its large color charge, the gluino is at the same time the most copiously produced SUSY particle at the LHC and a strong attractor in the RGEs for the stop and consequently for the Higgs. In most models, the gluino bounds are at or above the $\mathrm{TeV}$ level, which translates to a few percent tuning for split-family or even baryonic RPV models. In the latter case, the bounds on the gluino remain strong in part because the missing energy removed by RPV decay of the LSP is substituted by the leptons and missing energy that are produced from top production from the cascade decay: a natural spectrum should always contain light stops and sbottoms, making top production hard to avoid.

One may hope to relax the tuning in models of Dirac gauginos where the gluino can be out of reach by the LHC and does not contribute with log enhancement to the stop and Higgs masses because of supersoftness. Dirac gaugino models, however, have several challenges. First, the unification of gauge couplings is no longer automatic - the only experimental success of SUSY is lost. Second, the supersoft property is not ensured by a 


\begin{tabular}{|c|c|}
\hline Model & FT \\
\hline Mini-Split & $\lesssim 0.05 \%$ \\
MSSM & $0.3-1 \%$ \\
NMSSM & $2 \%$ \\
Split Families & $3 \%$ \\
bRPV & $2-3 \%$ \\
Dirac Gauginos & $(\lesssim 1 \%)$. \\
\hline
\end{tabular}

Table 1. Summary of the level of fine-tuning in the representative models studied in this paper compared to the 'unnatural' Mini-Split models [16, 89].

symmetry. On the contrary, in all known calculable models, supersoftness is badly broken and has to be recovered by cancellation, which makes the tuning below the $1 \%$ level. Successful model building with Dirac gauginos also has to deal with large RG corrections which may produce tachyons or big hierarchies in the spectrum that bring additional tension between naturalness and LHC bounds. Unless all these issues are addressed in a natural manner, one cannot claim that Dirac gauginos are a solution to tuning in SUSY.

In conclusion, while going beyond the MSSM may ameliorate naturalness, the improvement seems mild and only bring the tuning from $0.5-1 \%$ to the few percent level. Considering the required efforts in model building and the increased complexity of the resulting models it is natural to wonder whether such improvements are real. Whenever naturalness is ameliorated with an increase in complexity, there are hidden "unspeakable" tunings that are not captured by the standard formula but should be taken into account - every ingredient added and every parameter chosen to hide SUSY from us should in principle be paid in the tuning bill. Furthermore, since these types of tunings exist only to explain the absence of signals, they cannot even be explained with anthropic arguments and for this reason are qualitatively different, and worse, than the usual tuning of the EW scale.

There are several assumptions that come into the work presented above and warrant further discussion:

- We use the same measure of tuning and assume gauge mediated SUSY breaking (GMSB) for all the models discussed in order to compute the tuning reliably and guarantee gauge coupling unification as well as a natural solution to the SUSY-flavor problem. The common setup also allows for a fair and robust comparison of the level of the tuning among the different models.

- In addition to the gauge mediated contributions at the messenger scale, we allow for extra contributions to the Higgs-stop sector as suggested by models that address the $\mu-B_{\mu}$ problem. Assuming GMSB allows us to lower the mediation scale of the theory, reducing effects from RG running. On the other hand, the mediation scale cannot be pushed all the way down to the $\mathrm{TeV}$ scale without losing perturbativity, thus still leaving large RGE corrections to $m_{H_{u}}$ and $m_{\tilde{t}}$.

These limitations may be avoided in deconstructed models of gaugino mediation 
where a no-scale spectrum can be generated at low scales while still compatible with unification [90, 91]. Since the scalar boundary conditions vanish and the RGE effect is minimized by the low effective scale (which can even be below $10 \mathrm{TeV}$ ), the tuning in these models may be relaxed above $10 \%$. No-scale boundary conditions may however be troublesome because they lead to light right-handed sleptons - imposing the experimental bounds on these generically leads to stops above the $\mathrm{TeV}$ scale [92], reintroducing tuning. Also perturbative unification and the $\mu-B_{\mu}$ problem require extra model building in this class of models.

- Except for the MSSM example, we neglect any tuning required to fix the Higgs quartic coupling to its experimental value, and instead focus only on the tuning coming from the direct experimental bounds on the superpartners. Most known models to raise the Higgs mass are not completely natural and produce extra contributions to the tuning. Similarly, we neglect possible tuning hidden in the UV model addressing the $\mu-B_{\mu}$ problem and in the SUSY breaking sector generating the right gauge mediated setup. From this point of view the overall values of the tunings in table 1 should be interpreted as an optimistic upper bound to the actual amount of tuning.

- We did not consider combining models (e.g. split families or Dirac gauginos in bRPV). Even though such tricks may improve the canonical tuning, the increase in complexity (for the mere purpose of hiding SUSY from experimental searches) corresponds to an increase of tuning in theory space. While it is reasonable to expect that a property of the theory makes SUSY harder to be found, it is difficult to believe that this is achieved by combinations of effects acting coherently. Such a conspiracy would not be different from the canonical tuning.

- It is clear that several of the experimental bounds we present are model dependent or rely on simplified models. In the case of simplified models, unless the branching ratios through the specific channels are significantly modified, the bounds may be only slightly relaxed and do not make a difference in the tuning. In the case of bRPV, where we have considered specific spectra when applying the experimental analysis, we again do not expect a significant change when other models are considered. Since natural SUSY spectra should include light stops and higgsinos, we generically expect top production which guarantees leptons and missing energy from the $W \mathrm{~s}$ and $Z \mathrm{~s}$ produced in cascade decays.

Hiding SUSY at the LHC is very different from, for example, suppressing SUSY contributions to flavor observables. In order to suppress flavor violation, it is relatively easy to arrange a gauge-mediated model to give universal squark masses - any non-universality generated by RG flow is proportional to the yukawa couplings themselves so it is automatically minimally flavor violating. In contrast, there is no corresponding symmetry for "hiding" at the LHC: placing superpartners just at the right masses with just the right mass splittings is, by definition, not an RG-invariant statement and these spectra require very special boundary conditions in the UV. The associated tuning is exacerbated by the 
presence of IR fixed relations that tend to pull the spectrum away from the one required to hide SUSY. Such tuning is qualitatively worse than the standard tuning of the EW scale as it cannot even be explained anthropically. Models of this kind include compressed SUSY spectra where all jets or leptons are very soft or bRPV spectra which are squeezed to reduce top production.

We thus find that LHC searches for colored sparticles are pushing SUSY in the regime of percent level tuning, despite model-building efforts to explain the absence of sparticles at the LHC. The existence of tuning in the EW scale is by now a fact. What is still under debate is whether such a tuning may be interpreted as an accidental cancellation in the fundamental theory, which may still explain the smallness of the EW scale dynamically, or if it is the first signal that the smallness of the EW scale is the result of environmental selection, which does not require the presence of new light states. While the level of tuning required to distinguish the two possibilities is quite subjective, the numbers in table 1 should start making us quite uncomfortable with the first option.

Apart from the cosmological constant problem, this is the first time that effective field theory estimates fail so dramatically. Examples from QCD and nuclear physics (such as the size of the scattering length of certain di-nucleon systems) are sometimes used as arguments for the plausibility of percent-level tunings in nature. However, we do not think that such arguments can be used in support of naturalness for the EW scale. First, the examples referred above are often affected by QCD uncertainties, which makes a fair estimate of the tuning hard. Second, even accepting the most pessimistic estimates (which are at the few percent level), tunings appear only after having looked at many QCD observables and its relevance is washed out after taking into account the look-elsewhere effect. Nobody would be surprised if, after having discovered SUSY and measured its spectrum, one of the observables would appear to be tuned at the few percent level. On the contrary, finding a gap above the EW-scale appear as surprising as it would have been to find a gap between the pion and the other QCD resonances which is bigger than the natural one derived from the $\pi^{+}-\pi^{0}$ mass difference.

Percent cancellations are beyond the threshold of our tolerance and make us wonder: will a discovery of SUSY particles in the second run of the LHC be a true triumph of naturalness or a confirmation of its failure?

\section{Acknowledgments}

We warmly thank Savas Dimopoulos and Tony Gherghetta for collaboration in the early stage of this paper and for their valuable feedback throughout the completion of the work. We are also grateful to Tim Cohen, Nathaniel Craig, Mark Goodsell, Sonia El Hedri, Kiel Howe, David E. Kaplan, John March-Russell, Riccardo Rattazzi, Prashant Saraswat, Yuri Shirman, and Florian Staub for many useful comments and discussions.

We thank the CERN Theory Group for their hospitality during the completion of this work, and we also thank the Galileo Galilei Institute for Theoretical Physics for the hospitality and the INFN for partial support during the completion of this work. We 
acknowledge the help from FarmShare, Stanford's shared computing environment, which made MC event generation and analysis possible.

This work was supported in part by ERC grant BSMOXFORD no. 228169. MB is supported in part by the NSF Graduate Research Fellowship under Grant No. DGE1147470. XH is supported in part by the Stanford Humanities and Sciences Graduate Fellowship.

\section{A Taking into account correlations between different sources of tuning}

For the computation of the tuning in the different models we use the definitions in eqs. (1.1) and (1.2). Unlike in most of the literature where the tuning is computed taking only into account the dominant contribution in the sum in eq. (1.1), we include all the contributions in quadrature in order to reproduce the expected growth of the tuning with the square root of the number of parameters when they equally contribute to a given observable.

For the reasons explained in section 1.1, for the tuning of the EW vev we always use the Higgs formula

$$
m_{h}^{2}=-2\left(|\mu|^{2}+m_{H_{u}}^{2}\right),
$$

which gives a fair estimate of the tuning in all cases. The dependence on the fundamental parameters enters through $m_{H_{u}}^{2}$. In particular we consider the dependence with respect to: the $\mu$ term, the gluino mass at the messenger scale, the corrections to the gauge mediated boundary values of $m_{H_{u}}^{2}$ and $m_{\tilde{t}}^{2}$ at the messenger scale and other high-scale parameters the models may depend on, such as $A$-terms in the MSSM model or the extra messenger sector in the split-family model.

Unless specified differently, the total tuning includes the tuning against the EW vev and the stop mass. Since these two quantities depend on the same set of UV parameters they are not completely independent and we have to make sure not to overestimate the tuning. ${ }^{12}$ For this purpose we employ the following formula for the total tuning:

$$
\mathrm{FT}=\operatorname{Min}\left[\operatorname{Min}\left(1, \mathrm{FT}_{m_{h}^{2}}\right) \operatorname{Min}\left(1, \frac{\mathrm{FT}_{m_{\tilde{t}}^{2}}}{\sin \theta}\right), \operatorname{Min}\left(1, \mathrm{FT}_{m_{\tilde{t}}^{2}}\right) \operatorname{Min}\left(1, \frac{\mathrm{FT}_{m_{h}^{2}}}{\sin \theta}\right)\right],
$$

where

$$
\sin \theta \equiv \frac{\left|\vec{v}_{m_{h}^{2}} \times \vec{v}_{m_{\tilde{t}}^{2}}\right|}{\left|\vec{v}_{m_{h}^{2}}\right|\left|\vec{v}_{m_{\tilde{t}}^{2}}\right|}, \quad v_{x}^{i} \equiv \frac{\partial \log x}{\partial \log a_{i}}, \quad \mathrm{FT}_{x} \equiv \frac{1}{\left|\vec{v}_{x}\right|} .
$$

This formula interpolates between

$$
\mathrm{FT}=\mathrm{FT}_{m_{h}^{2}} \cdot \mathrm{FT}_{m_{\tilde{t}}^{2}}
$$

when the two tuning are completely independent $(\sin \theta=1)$ and

$$
\mathrm{FT}=\operatorname{Min}\left[\mathrm{FT}_{m_{h}^{2}}, \mathrm{FT}_{m_{\tilde{t}}^{2}}\right],
$$

when they are completely dependent $(\sin \theta=0)$.

\footnotetext{
${ }^{12}$ We thank David Pinner and Josh Ruderman for raising this potential issue.
} 
Having a large correlation implies that by tuning the stop to be light against the gluino, the Higgs mass is automatically relieved by the largest contribution and does not require additional tuning. For high-scale messengers this is not the case because for most of the running the stop mass is large due to the gluino attraction and gives a significant contribution to the $m_{H_{u}}^{2}$. The correlation is also not important in low-scale gauge mediated models because of the extra contributions to $m_{H_{u}}^{2}$ and $m_{\tilde{t}}^{2}$ at the messenger scale. In fact in all our examples we checked that the effect from the correlation is never significant and treating the two tunings as independent gives a good estimate of the overall tuning.

\section{B Split families details}

Here we explain the split family model in more detail. All particle content receives soft SUSY breaking masses via gauge mediation from vector-like $\mathbf{5}+\overline{\mathbf{5}}$ messenger pairs $\left(D^{\prime}+\right.$ $\left.\bar{D}^{\prime}, L^{\prime}+\bar{L}^{\prime}\right)$. The first two generations receive additional soft SUSY breaking masses through a pair of messengers $N+\bar{N}$ which are charged only under the new $\mathrm{U}(1)^{\prime}$. The messengers communicate the effects of SUSY breaking parameterized by spurions $X_{D}=m_{D}+F \theta^{2}$ and $X_{N}=m_{N}+F \theta^{2}$ :

$$
\mathcal{W}_{\text {mess }}=X_{D}\left(D^{\prime} \bar{D}^{\prime}+L^{\prime} \bar{L}^{\prime}\right)+X_{N} N \bar{N}
$$

Since the third generation is not charged under $\mathrm{U}(1)^{\prime}$, the flavor structure of our model is non-trivial. The Yukawa terms cannot contain mixing between the first two and third generations:

$$
\begin{gathered}
\mathcal{W}_{\mathrm{MSSM}}=y_{i j}^{u} H_{u} Q_{i} U_{j}+y_{3}^{u} H_{u} Q_{3} U_{3}+y_{i j}^{d} H_{d} Q_{i} D_{j}+y_{3}^{d} H_{d} Q_{3} D_{3}+ \\
y_{i j}^{e} H_{d} L_{i} E_{j}+y_{3}^{e} H_{d} L_{3} E_{3}+\mu H_{u} H_{d} \quad(i, j=1,2)
\end{gathered}
$$

The spontaneous breaking of $\mathrm{U}(1)^{\prime}$ generates the mixing between $D_{3}$ and messenger $\bar{D}^{\prime \prime}$ from superpotential

$$
\mathcal{W} \supset \lambda_{i} H_{d} Q_{i} D^{\prime \prime}+\Phi \bar{D}^{\prime \prime} D_{3}+m_{D^{\prime \prime}} D^{\prime \prime} \bar{D}^{\prime \prime}+V(\Phi)_{\mathrm{U}(1)^{\prime} \text {-breaking }} \quad(i=1,2) .
$$

Integrating out the messengers $D^{\prime \prime}$ and $\bar{D}^{\prime \prime}$ generates the required Yukawa mixing:

$$
\mathcal{W}_{\text {mixing }}=\lambda_{i} \frac{\langle\Phi\rangle}{m_{D^{\prime \prime}}} H_{d} Q_{i} D_{3},
$$

with $\lambda_{i} \sim \mathcal{O}(1)$. Note that messengers $D^{\prime \prime}, \bar{D}^{\prime \prime}$ cannot couple to the SUSY breaking spurions; otherwise unacceptably large $A$-terms and off-diagonal soft masses will be generated, resulting in large flavor violation. Forbidding this coupling can be accomplished by assigning appropriate $\mathrm{U}(1)^{\prime}$ charges to $D^{\prime}, D^{\prime \prime}$, and the SUSY breaking spurions.

To summarize, there are 4 scales: $m_{D^{\prime \prime}}>m_{D}>m_{N} \gtrsim\langle\Phi\rangle$. The ratio $\langle\Phi\rangle / m_{D^{\prime \prime}}$ sets the magnitude of the Yukawa mixing between the third and first/second generations; $m_{D} / m_{N}$ sets the mass splitting between the sfermions. $m_{N}$ sets the scale of mediation to the first two generations. We take $m_{N} \gtrsim\langle\Phi\rangle$ so that the gauge mediation from $\mathrm{U}(1)^{\prime}$ gauge bosons is not suppressed. 
Contribution to flavor observables. The superpotential given in eqs. (B.2) and (B.4) is not in field basis convenient for comparison with the Standard Model Yukawa terms; in particular, there is no $Q_{3} D_{1}$ or $Q_{3} D_{2}$ mixing. Therefore to study the flavor structure, we need to match our parameters $\left(y_{i j}^{u}, y_{i j}^{d}, y_{3}^{u}, y_{3}^{d}, \lambda_{i}\right)$ to the Yukawas and CKM elements and rotate to the new basis accordingly. Only 10 (real) parameters are physical, ${ }^{13}$ corresponding exactly to the 10 degrees of freedom (6 quark masses, 3 CKM angles, $1 \mathrm{CP}$ phase) in the Standard Model. The field rotation disturbs the flavor-diagonal nature of gauge mediation and therefore can induce potentially dangerous flavor violation (see e.g. [93-95]).

In our toy model, however, such effects are small; the smallness can be understood best in a basis where the up sector is diagonal. In this basis, the three $D_{i}$ need to be rotated from a basis where the down sector mass matrix is 0 in the $(3,1)$ and $(3,2)$ entries, to a basis where the mass matrix is $V_{\mathrm{CKM}} D$, where $D$ is a diagonal matrix with $y_{d}, y_{s}$, and $y_{b}$ on the diagonal. Numerically, the $(3,1)$ and $(3,2)$ entries of $V_{\mathrm{CKM}} D$ are much smaller than the other entries, therefore requiring only a small rotation away from the gauge mediation basis. We verified with SUSY_FLAVOR v2.02 [96] that the additional contribution to sensitive flavor observables agree with the SM prediction within theoretical error. We checked that already for first two generation squarks at $3 \mathrm{TeV}$ and above the corrections to $\varepsilon_{K}$ and other flavor observables are negligible.

\section{RPV details}

Here we discuss in more detail the 'toy model' presented in section 3.2; in the following subsections we provide further details of limits on UDD couplings and discuss our procedure for event generation and recasting of experimental analyses to set limits on the model.

In order to preserve the success of unification in the MSSM while introducing baryon but not lepton number violation, we use a solution already in place for the Higgs doublettriplet splitting problem: orbifold GUTs [97]. To generate the RPV couplings, consider an orbifold with $\mathrm{SU}(5)$ in the bulk, and $\mathrm{SU}(3) \times \mathrm{SU}(2) \times \mathrm{U}(1)$ on the IR brane $(z=0)$. The matter content has varying profiles in the bulk. The Higgses are on the IR brane, leading to a different yukawa hierarchy in the up and down sectors,

$$
y_{i}^{u}=\left(\left.Q_{i}\right|_{z=0}\right)^{2}=\left(\left.U_{i}\right|_{z=0}\right)^{2} \quad \text { while } \quad y_{i}^{d}=\left(\left.Q_{i}\right|_{z=0}\right)\left(\left.D_{i}\right|_{z=0}\right) .
$$

On the IR brane we introduce heavy $D^{\prime}$ and $\Phi$ fields charged under baryon number and with $B$-invariant superpotential couplings

$$
\left.\mathcal{W}_{R P V}\right|_{z=0} \supset \kappa_{i} U_{i} D^{\prime} D^{\prime}+\bar{\kappa}_{j} \Phi D_{j} \bar{D}^{\prime}+M_{D} D^{\prime} \bar{D}^{\prime},
$$

the baryon number is then broken spontaneously after the field $\Phi$ gets a vev. ${ }^{14}$ Since B-breaking is introduced on the IR brane, leptonic partners of down-type fields are not

\footnotetext{
${ }^{13}\left(y_{i j}^{u}, y_{i j}^{d}, y_{3}^{u}, y_{3}^{d}, \lambda_{i}\right)$ contains 12 complex, or 24 real parameters. Unitary rotations of the first two generations render $4 \times 3$ of them unphysical, and 3 additional phases can be absorbed into the third generation fields. Adding back the overall conserved baryon number, there are $24-12-3+1=10$ real physical parameters left.

${ }^{14}$ There may be a light axion that is associated with the breaking of baryon number. Its exact mass depends on the details of the UV model and its axion decay constant is close to the GUT scale, so it doesn't introduce significant experimental constraints.
} 


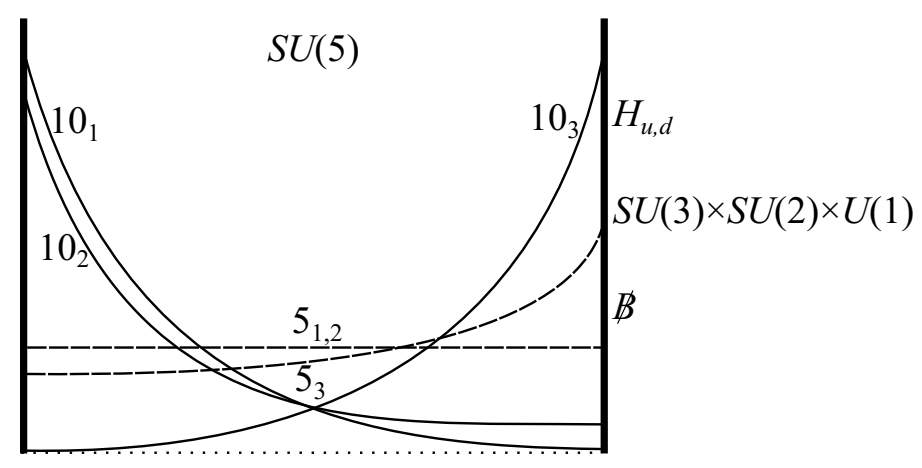

Figure 7. Toy orbifold model for bRPV.

required to preserve unification, and do not induce lepton number violation. Integrating out the $D^{\prime}$ field then gives a baryon number violating term,

$$
\mathcal{W}_{\not B}=\left.\frac{\langle\Phi\rangle^{2}}{M_{D}^{2}} \kappa_{i} \bar{\kappa}_{j} \bar{\kappa}_{k} U_{i} D_{j} D_{k}\right|_{z=0}
$$

we take $\kappa_{i} \sim \bar{\kappa}_{j} \sim \mathcal{O}(1)$, resulting in a predictive pattern of RPV couplings,

$$
\left.\lambda_{i j k}^{\prime \prime} \simeq \frac{\langle\Phi\rangle^{2}}{M_{D}^{2}}\left(U_{i} D_{j} D_{k}\right)\right|_{z=0}=\frac{\langle\Phi\rangle^{2}}{M_{D}^{2}} \sqrt{y_{i}^{u}} \frac{y_{j}^{d}}{\sqrt{y_{j}^{u}}} \frac{y_{k}^{d}}{\sqrt{y_{k}^{u}}} .
$$

SUSY breaking is mediated via doublet-triplet split gauge mediation such that the ratio of the wino to the gluino mass may be allowed to vary; we find, however, that a heavy wino does not significantly reduce experimental bounds at the expense of increased tuning from an additional parameter.

RPV coupling constraints. One of the most constraining limits on $\lambda_{i j k}^{\prime \prime}$ comes from $n$ $\bar{n}$ oscillations: the tree level process puts limits on $\lambda_{11 k}^{\prime \prime}$. Because of the left-right insertions in this process, a more constraining limit on $\lambda_{112}^{\prime \prime}$ results from decays ${ }^{16} \mathrm{O} \rightarrow{ }^{14} \mathrm{CK}^{+} \mathrm{K}^{+}$, through $n-\Xi$ oscillations. For a decay lifetime $\tau>10^{31} \mathrm{~s}$, the limit is [61, 63],

$$
\lambda_{112}^{\prime \prime} \lesssim 10^{-5}\left(\frac{m_{\tilde{g}}}{600 \mathrm{GeV}}\right)^{1 / 2}\left(\frac{m_{\tilde{d}_{R}}}{600 \mathrm{GeV}}\right)^{2}\left(\frac{10^{-6} \mathrm{GeV}^{6}}{\left\langle n\left|u_{r} d_{r} u_{r} d_{r} s_{r} s_{r}\right| \Xi\right\rangle}\right)^{1 / 2} .
$$

These limits are subject to large nuclear uncertainties; in addition, $n-\bar{n}$ oscillations place a limit of the same order of magnitude $\lambda_{121}^{\prime \prime}$ where one of the quarks participating in the process is a strange quark which makes up about $10 \%$ of the neutron at zero momentum.

Loop-level $n-\bar{n}$ oscillations also put a constraint on third generation RPV couplings $\lambda_{3 j k}^{\prime \prime}$,

$$
\begin{aligned}
& \lambda_{321}^{\prime \prime}<0.15\left(\frac{M_{2}}{600 \mathrm{GeV}}\right)^{1 / 2}\left(\frac{m_{\tilde{q}_{L}}}{200 \mathrm{GeV}}\right)^{2}\left(\frac{m_{\tilde{q}_{R}}}{200 \mathrm{GeV}}\right)^{2}\left(\frac{(600 \mathrm{GeV})^{2}}{A_{L R}^{t} A_{L R}^{s}}\right)\left(\frac{10^{-4} \mathrm{GeV}^{6}}{|\psi(0)|^{4}}\right)^{1 / 2}, \\
& \lambda_{331}^{\prime \prime}<0.3\left(\frac{M_{2}}{600 \mathrm{GeV}}\right)^{1 / 2}\left(\frac{m_{\tilde{q}_{L}}}{600 \mathrm{GeV}}\right)^{2}\left(\frac{m_{\tilde{q}_{R}}}{600 \mathrm{GeV}}\right)^{2}\left(\frac{(600 \mathrm{GeV})^{2}}{A_{L R}^{t} A_{L R}^{b}}\right)\left(\frac{10^{-4} \mathrm{GeV}^{6}}{|\psi(0)|^{4}}\right)^{1 / 2} .
\end{aligned}
$$




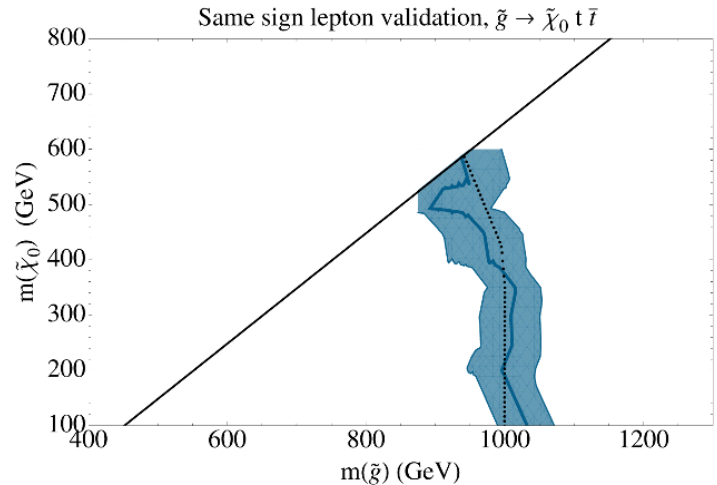

(a)

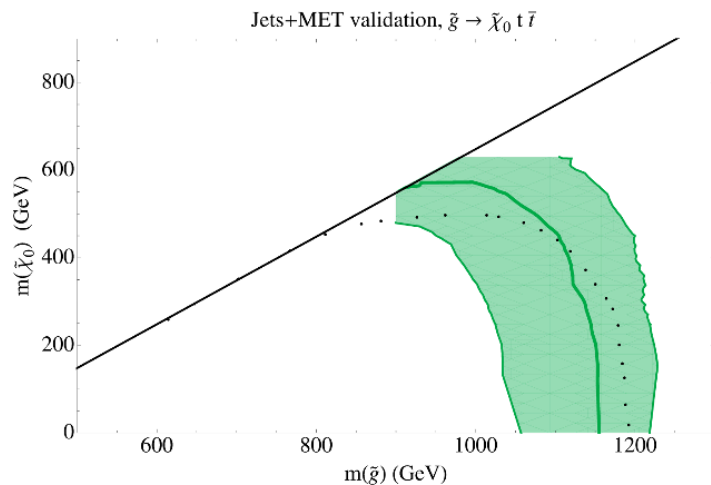

(b)

Figure 8. Validation of search implementations for (a) CMS SS dileptons and b-jets [67] and (b) ATLAS 7-10 jets and MET [65]. The experimental limit is indicated with the dotted line, and our limit has been rescaled to match the observed bound by (a) $2.4 \pm 0.6$ and (b) $1.1_{-0.5}^{+1.6}$. The leptonic search matches quite well after the rescaling, which may be a result of overestimating lepton efficiencies; the jet search is an overestimate in the squeezed region, and we take this into account by introducing a large uncertainty.

There are also limits on products of $\lambda^{\prime \prime}[45]$; the most constraining limits result from rare $B$ decays and $K-\bar{K}$ oscillations, and provide limits on second-generation couplings, but are less constraining for first and third generations.

$$
\left|\lambda_{i j k}^{\prime \prime} \lambda_{i j k}^{\prime \prime}\right|^{1 / 2} \lesssim 10^{-1}-10^{-2}
$$

Taking into account all of the above constraints, the strongest limits on the model which has a square root of yukawa coupling hierarchy come from the first generation, $\lambda_{1 j k}^{\prime \prime} \lesssim 10^{-5}$ for squarks at $600 \mathrm{GeV}$. This is only an order of magnitude bound due to the large nuclear uncertainties in this limit; we pick the maximum allowed RPV couplings to study the phenomenology of the model (3.7).

Simulation details. Given that the current LHC limits as applied to RPV models are limited, we recast the most relevant searches to set limits on the model described in section 3.2. We summarize our procedure below. Our bounds tend to be stronger than other re-castings of the limits which have been done in the literature; this is due either to older searches with smaller data sets [49, 59], or assumptions of restricted production channels $[98,99]$. We find, for example, that our same-sign lepton search recasting sets a limit of $M_{3}>850 \mathrm{GeV}$ for the gluino pair-production channel, which matches the limit derived by other authors [99].

We create a grid in the gluino-stop plane in order to study the constraints. We simulate the hard scattering LHC processes at $\sqrt{s}=8 \mathrm{TeV}$ for the leading squark-squark, squarkgluino, and gluino-gluino production channels MadGraph5 v1.5.7 [100]. For each parameter point we compute the low-energy spectrum using SOFTSUSY v3.3.4 [101]; we calculate the branching ratios, including the RPV couplings according to the pattern in eq. (3.7) and assuming a Higgs mass of $m_{h}=125 \mathrm{GeV}$ using BRIDGE v2.24 [102] and MadGraph5. 
We confirm the branching ratios with SDECAY v1.3 [103] and find agreement between the two methods within 5\%. We compute NLO cross-sections for the processes in Prospino v2.1 [104] and decay and shower the complete spectrum using Pythia v8.175 [105]. Finally, we use the detector simulator Delphes v3.0.5 [106] with jet radius, and lepton and b-tag efficiencies as specified in the corresponding CMS and ATLAS analyses [65, 67].

To check our analysis against experimental searches, we use our pipeline of MadGraph5, Pythia, and Delphes on an example model of $p p \rightarrow \tilde{g} \tilde{g}, \tilde{g} \rightarrow t \bar{t} \tilde{\chi}^{0}$ through an off-shell stop. We find that the limits of our analysis are in good agreement with those set by the experimental collaborations, and we rescale our efficiencies by factor of $2.4 \pm 0.6$ and $1.1_{-0.5}^{+1.6}$ in the leptonic search and the jets search, respectively, to match the experimental results (see figure 8), which introduces an uncertainty in our limits of $150 \mathrm{GeV}$ in the leptons and $200 \mathrm{GeV}$ in the jet search. We use the same central values and uncertainty bands as in the validation plots to set limits on the RPV spectrum in figure 5 . It would be very interesting to see an experimental analysis of these searches in the context of RPV, as well as a wider range of experimental recastings for simplified models with RPV decays.

Open Access. This article is distributed under the terms of the Creative Commons Attribution License (CC-BY 4.0), which permits any use, distribution and reproduction in any medium, provided the original author(s) and source are credited.

\section{References}

[1] S. Dimopoulos, S. Raby and F. Wilczek, Supersymmetry and the Scale of Unification, Phys. Rev. D 24 (1981) 1681 [INSPIRE].

[2] S. Dimopoulos and H. Georgi, Softly Broken Supersymmetry and SU(5), Nucl. Phys. B 193 (1981) 150 [INSPIRE].

[3] N. Arkani-Hamed and S. Dimopoulos, Supersymmetric unification without low energy supersymmetry and signatures for fine-tuning at the LHC, JHEP 06 (2005) 073 [hep-th/0405159] [INSPIRE].

[4] R. Barbieri and G. Giudice, Upper Bounds on Supersymmetric Particle Masses, Nucl. Phys. B 306 (1988) 63 [INSPIRE].

[5] CMS collaboration, Observation of a new boson with mass near $125 \mathrm{GeV}$ in pp collisions at $\sqrt{s}=7$ and $8 \mathrm{TeV}$, JHEP 06 (2013) 081 [arXiv:1303.4571] [INSPIRE].

[6] ATLAS collaboration, Measurements of Higgs boson production and couplings in diboson final states with the ATLAS detector at the LHC, Phys. Lett. B 726 (2013) 88 [arXiv: 1307.1427] [INSPIRE].

[7] N. Craig, J. Galloway and S. Thomas, Searching for Signs of the Second Higgs Doublet, arXiv:1305.2424 [INSPIRE].

[8] A. Arvanitaki and G. Villadoro, A Non Standard Model Higgs at the LHC as a Sign of Naturalness, JHEP 02 (2012) 144 [arXiv:1112.4835] [INSPIRE].

[9] J.L. Feng, K.T. Matchev and T. Moroi, Focus points and naturalness in supersymmetry, Phys. Rev. D 61 (2000) 075005 [hep-ph/9909334] [INSPIRE].

[10] D. Horton and G. Ross, Naturalness and Focus Points with Non-Universal Gaugino Masses, Nucl. Phys. B 830 (2010) 221 [arXiv:0908.0857] [inSPIRE]. 
[11] G. Giudice and R. Rattazzi, Theories with gauge mediated supersymmetry breaking, Phys. Rept. 322 (1999) 419 [hep-ph/9801271] [INSPIRE].

[12] G.F. Giudice, H.D. Kim and R. Rattazzi, Natural mu and B mu in gauge mediation, Phys. Lett. B 660 (2008) 545 [arXiv: 0711 .4448] [InSPIRE].

[13] A. De Simone, R. Franceschini, G.F. Giudice, D. Pappadopulo and R. Rattazzi, Lopsided Gauge Mediation, JHEP 05 (2011) 112 [arXiv:1103.6033] [INSPIRE].

[14] ATLAS collaboration, Search for squarks and gluinos with the ATLAS detector in final states with jets and missing transverse momentum and $20.3 \mathrm{fb}^{-1}$ of $\sqrt{\mathrm{s}}=8 \mathrm{TeV}$ proton-proton collision data, ATLAS-CONF-2013-047 (2013).

[15] G.F. Giudice and A. Strumia, Probing High-Scale and Split Supersymmetry with Higgs Mass Measurements, Nucl. Phys. B 858 (2012) 63 [arXiv:1108.6077] [INSPIRE].

[16] A. Arvanitaki, N. Craig, S. Dimopoulos and G. Villadoro, Mini-Split, JHEP 02 (2013) 126 [arXiv:1210.0555] [INSPIRE].

[17] U. Ellwanger, C. Hugonie and A.M. Teixeira, The Next-to-Minimal Supersymmetric Standard Model, Phys. Rept. 496 (2010) 1 [arXiv:0910.1785] [InSPIRE].

[18] P. Batra, A. Delgado, D.E. Kaplan and T.M. Tait, The Higgs mass bound in gauge extensions of the minimal supersymmetric standard model, JHEP 02 (2004) 043 [hep-ph/0309149] [INSPIRE].

[19] R. Harnik, G.D. Kribs, D.T. Larson and H. Murayama, The Minimal supersymmetric fat Higgs model, Phys. Rev. D 70 (2004) 015002 [hep-ph/0311349] [INSPIRE].

[20] R. Barbieri, L.J. Hall, Y. Nomura and V.S. Rychkov, Supersymmetry without a Light Higgs Boson, Phys. Rev. D 75 (2007) 035007 [hep-ph/0607332] [InSPIRE].

[21] S. Dimopoulos and G. Giudice, Naturalness constraints in supersymmetric theories with nonuniversal soft terms, Phys. Lett. B 357 (1995) 573 [hep-ph/9507282] [INSPIRE].

[22] A.G. Cohen, D. Kaplan and A. Nelson, The More minimal supersymmetric standard model, Phys. Lett. B 388 (1996) 588 [hep-ph/9607394] [INSPIRE].

[23] A. Pomarol and D. Tommasini, Horizontal symmetries for the supersymmetric flavor problem, Nucl. Phys. B 466 (1996) 3 [hep-ph/9507462] [INSPIRE].

[24] ATLAS collaboration, Search for strong production of supersymmetric particles in final states with missing transverse momentum and at least three b-jets using $20.1 \mathrm{fb}^{-1}$ of $\mathrm{pp}$ collisions at $\sqrt{s}=8 \mathrm{TeV}$ with the ATLAS Detector., ATLAS-CONF-2013-061 (2013).

[25] ATLAS collaboration, Search for direct production of the top squark in the all-hadronic ttbar + etmiss final state in $21 \mathrm{fb}-1$ of p-pcollisions at $\sqrt{s}=8 \mathrm{TeV}$ with the ATLAS detector, ATLAS-CONF-2013-024 (2013).

[26] CMS collaboration, Search for supersymmetry using razor variables in events with b-jets in pp collisions at 8 tev, CMS-PAS-SUS-13-004.

[27] CMS collaboration, Search for top-squark pair production in the single-lepton final state in pp collisions at $\sqrt{s}=8 \mathrm{TeV}$, CERN-PH-EP-2013-148 (2013) [CMS-SUS-13-011-003].

[28] CMS collaboration, Search for SUSY Partners of Top and Higgs Using Diphoton Higgs Decays, CMS-PAS-SUS-13-014.

[29] A. Delgado, G.F. Giudice, G. Isidori, M. Pierini and A. Strumia, The light stop window, Eur. Phys. J. C 73 (2013) 2370 [arXiv:1212.6847] [InSPIRE]. 
[30] CMS collaboration, Search for electroweak production of charginos, neutralinos and sleptons using leptonic final states in pp collisions at $8 \mathrm{TeV}$, CMS-PAS-SUS-13-006.

[31] P. Meade, M. Reece and D. Shih, Prompt Decays of General Neutralino NLSPs at the Tevatron, JHEP 05 (2010) 105 [arXiv:0911.4130] [INSPIRE].

[32] K. Howe and P. Saraswat, Excess Higgs Production in Neutralino Decays, JHEP 10 (2012) 065 [arXiv: 1208.1542] [INSPIRE].

[33] ATLAS collaboration, Search for chargino and neutralino production in final states with one lepton, two b-jets consistent with a Higgs boson and missing transverse momentum with the ATLAS detector in 20.3 $\mathrm{fb}^{-1}$ of $\sqrt{\mathrm{s}}=8 \mathrm{TeV}$ pp collisions, ATLAS-CONF-2013-093 (2013).

[34] LEP2 SUSY Working Group, Combined lep selectron/smuon/stau results, 183-208 GeV, LEPSUSYWG/04-01.1 (2004).

[35] ATLAS collaboration, Search for Supersymmetry in Events with Large Missing Transverse Momentum, Jets and at Least One Tau Lepton in $21 \mathrm{fb}^{-1}$ of $\sqrt{s}=8$ TeV Proton-Proton Collision Data with the ATLAS Detector, ATLAS-CONF-2013-026 (2013).

[36] ATLAS collaboration, A search for heavy long-lived sleptons using $16 \mathrm{fb}^{-1}$ of pp collisions at $\sqrt{s}=8 \mathrm{TeV}$ with the ATLAS detector, ATLAS-CONF-2013-058 (2013).

[37] CMS collaboration, Searches for long-lived charged particles in pp collisions at $\sqrt{s}=7$ and 8 TeV, JHEP 07 (2013) 122 [arXiv: 1305. 0491] [INSPIRE].

[38] G. Dvali and A. Pomarol, Anomalous U(1) as a mediator of supersymmetry breaking, Phys. Rev. Lett. 77 (1996) 3728 [hep-ph/9607383] [INSPIRE].

[39] E. Hardy and J. March-Russell, Retrofitted Natural Supersymmetry from a U(1), JHEP 05 (2013) 120 [arXiv: 1302.5423] [INSPIRE].

[40] M. Gabella, T. Gherghetta and J. Giedt, A Gravity dual and LHC study of single-sector supersymmetry breaking, Phys. Rev. D 76 (2007) 055001 [arXiv:0704.3571] [InSPIRE].

[41] N. Craig, S. Dimopoulos and T. Gherghetta, Split families unified, JHEP 04 (2012) 116 [arXiv:1203.0572] [INSPIRE].

[42] N. Craig, D. Green and A. Katz, (De)Constructing a Natural and Flavorful Supersymmetric Standard Model, JHEP 07 (2011) 045 [arXiv: 1103.3708] [INSPIRE].

[43] N. Craig, M. McCullough and J. Thaler, Flavor Mediation Delivers Natural SUSY, JHEP 06 (2012) 046 [arXiv: 1203.1622] [INSPIRE].

[44] E. Hardy, Is Natural SUSY Natural?, JHEP 10 (2013) 133 [arXiv:1306.1534] [INSPIRE].

[45] R. Barbier et al., R-parity violating supersymmetry, Phys. Rept. 420 (2005) 1 [hep-ph/0406039] [INSPIRE].

[46] CMS collaboration,, Search for RPV supersymmetry with three or more leptons and b-tags, CMS-PAS-SUS-12-027.

[47] J.A. Evans and Y. Kats, LHC Coverage of RPV MSSM with Light Stops, JHEP 04 (2013) 028 [arXiv: 1209.0764] [INSPIRE].

[48] Z. Han, A. Katz, M. Son and B. Tweedie, Boosting Searches for Natural SUSY with RPV via Gluino Cascades, Phys. Rev. D 87 (2013) 075003 [arXiv:1211.4025] [InSPIRE].

[49] B. Allanach and B. Gripaios, Hide and Seek With Natural Supersymmetry at the LHC, JHEP 05 (2012) 062 [arXiv: 1202.6616] [INSPIRE].

[50] C. Brust, A. Katz and R. Sundrum, SUSY Stops at a Bump, JHEP 08 (2012) 059 [arXiv: 1206.2353] [INSPIRE]. 
[51] CMS collaboration, Search for three-jet resonances in pp collisions at $\sqrt{s}=7 \mathrm{TeV}$, Phys. Lett. B 718 (2012) 329 [arXiv: 1208.2931] [INSPIRE].

[52] ATLAS collaboration, Search for pair production of massive particles decaying into three quarks with the ATLAS detector in $\sqrt{s}=7 \mathrm{TeV}$ pp collisions at the LHC, JHEP 12 (2012) 086 [arXiv: 1210.4813] [INSPIRE].

[53] B. Bhattacherjee, J.L. Evans, M. Ibe, S. Matsumoto and T.T. Yanagida, Natural SUSY's Last Hope: R-parity Violation via UDD Operators, Phys. Rev. D 87 (2013) 115002 [arXiv: 1301.2336] [INSPIRE].

[54] R. Franceschini and R. Mohapatra, New Patterns of Natural R-Parity Violation with Supersymmetric Gauged Flavor, JHEP 04 (2013) 098 [arXiv:1301.3637] [INSPIRE].

[55] L. Di Luzio, M. Nardecchia and A. Romanino, A framework for baryonic R-parity violation in grand unified theories, Phys. Rev. D 88 (2013) 115008 [arXiv: 1305.7034] [INSPIRE].

[56] ALEPH collaboration, A. Heister et al., Search for supersymmetric particles with $R$ parity violating decays in $e^{+} e^{-}$collisions at $\sqrt{s}$ up to 209-GeV, Eur. Phys. J. C 31 (2003) 1 [hep-ex/0210014] [INSPIRE].

[57] ATLAS collaboration, Search for strongly produced superpartners in final states with two same sign leptons with the ATLAS detector using $21 \mathrm{fb}-1$ of proton-proton collisions at $\sqrt{s}=8 \mathrm{TeV}$., ATLAS-CONF-2013-007 (2013).

[58] ATLAS collaboration, Search for massive particles in multijet signatures with the ATLAS detector in $\sqrt{s}=8 \mathrm{TeV}$ pp collisions at the LHC, ATLAS-CONF-2013-091 (2013).

[59] M. Asano, K. Rolbiecki and K. Sakurai, Can R-parity violation hide vanilla supersymmetry at the LHC?, JHEP 01 (2013) 128 [arXiv:1209.5778] [INSPIRE].

[60] S. Dimopoulos and L.J. Hall, Baryogenesis at the MeV Era, Phys. Lett. B 196 (1987) 135 [INSPIRE].

[61] R. Barbieri and A. Masiero, Supersymmetric Models with Low-Energy Baryon Number Violation, Nucl. Phys. B 267 (1986) 679 [INSPIRE].

[62] Y. Cui and R. Sundrum, Baryogenesis for weakly interacting massive particles, Phys. Rev. D 87 (2013) 116013 [arXiv:1212.2973] [INSPIRE].

[63] J. Goity and M. Sher, Bounds on delta $B=1$ couplings in the supersymmetric standard model, Phys. Lett. B 346 (1995) 69 [Erratum ibid. B 385 (1996) 500] [hep-ph/9412208] [INSPIRE].

[64] CMS collaboration, Search for long-lived particles decaying to photons and missing energy in proton-proton collisions at $\sqrt{s}=7$ TeV, Phys. Lett. B 722 (2013) 273 [arXiv:1212.1838] [INSPIRE].

[65] ATLAS collaboration, Search for new phenomena using final states with large jet multiplicities and missing transverse momentum with ATLAS in $20 \mathrm{fb}^{-1}$ of $\sqrt{\mathrm{s}}=8 \mathrm{TeV}$ proton-proton collisions, ATLAS-CONF-2013-054 (2013).

[66] CMS collaboration, Search for supersymmetry in hadronic final states with missing transverse energy using the variables AlphaT and b-quark multiplicity in pp collisions at 8 TeV, Eur. Phys. J. C 73 (2013) 2568 [arXiv:1303.2985] [InSPIRE].

[67] CMS collaboration, Search for new physics in events with same-sign dileptons and $b$ jets in pp collisions at $\sqrt{s}=8 \mathrm{TeV}$, JHEP 03 (2013) 037 [Erratum ibid. 1307 (2013) 041] [arXiv:1212.6194] [INSPIRE]. 
[68] CMS collaboration, Search for new physics in events with same-sign dileptons and jets in pp collisions at $8 \mathrm{TeV}$, CMS-PAS-SUS-13-013.

[69] P. Fayet, Massive gluinos, Phys. Lett. B 78 (1978) 417 [InSPIRE].

[70] J. Polchinski and L. Susskind, Breaking of Supersymmetry at Intermediate-Energy, Phys. Rev. D 26 (1982) 3661 [INSPIRE].

[71] L. Hall and L. Randall, U(1)-R symmetric supersymmetry, Nucl. Phys. B 352 (1991) 289 [INSPIRE].

[72] P.J. Fox, A.E. Nelson and N. Weiner, Dirac gaugino masses and supersoft supersymmetry breaking, JHEP 08 (2002) 035 [hep-ph/0206096] [INSPIRE].

[73] A.E. Nelson, N. Rius, V. Sanz and M. Ünsal, The Minimal supersymmetric model without a mu term, JHEP 08 (2002) 039 [hep-ph/0206102] [INSPIRE].

[74] I. Antoniadis, K. Benakli, A. Delgado and M. Quirós, A New gauge mediation theory, Adv. Stud. Theor. Phys. 2 (2008) 645 [hep-ph/0610265] [INSPIRE].

[75] S.D.L. Amigo, A.E. Blechman, P.J. Fox and E. Poppitz, R-symmetric gauge mediation, JHEP 01 (2009) 018 [arXiv:0809.1112] [INSPIRE].

[76] G.D. Kribs and A. Martin, Supersoft Supersymmetry is Super-Safe, Phys. Rev. D 85 (2012) 115014 [arXiv: 1203.4821] [InSPIRE].

[77] M. Heikinheimo, M. Kellerstein and V. Sanz, How Many Supersymmetries?, JHEP 04 (2012) 043 [arXiv: 1111.4322] [INSPIRE].

[78] G.D. Kribs, E. Poppitz and N. Weiner, Flavor in supersymmetry with an extended R-symmetry, Phys. Rev. D 78 (2008) 055010 [arXiv:0712.2039] [INSPIRE].

[79] K. Benakli and M. Goodsell, Dirac Gauginos, Gauge Mediation and Unification, Nucl. Phys. B 840 (2010) 1 [arXiv:1003.4957] [InSPIRE].

[80] CMS collaboration, Search for New Physics in the Multijets and Missing Momentum Final State in Proton-Proton Collisions at 8 TeV, CMS-PAS-SUS-13-012.

[81] CMS collaboration, Search for supersymmetry with the razor variables at CMS, CMS-PAS-SUS-12-005.

[82] CMS collaboration, Search for direct EWK production of SUSY particles in multilepton modes with 8 TeV data, CMS-PAS-SUS-12-022.

[83] K. Benakli and M. Goodsell, Dirac Gauginos in General Gauge Mediation, Nucl. Phys. B 816 (2009) 185 [arXiv:0811.4409] [INSPIRE].

[84] L.M. Carpenter, Dirac Gauginos, Negative Supertraces and Gauge Mediation, JHEP 09 (2012) 102 [arXiv:1007.0017] [InSPIRE].

[85] K. Benakli, M.D. Goodsell and F. Staub, Dirac Gauginos and the 125 GeV Higgs, JHEP 06 (2013) 073 [arXiv: 1211.0552] [INSPIRE].

[86] M.D. Goodsell, Two-loop RGEs with Dirac gaugino masses, JHEP 01 (2013) 066 [arXiv:1206.6697] [INSPIRE].

[87] S.P. Martin and M.T. Vaughn, Two loop renormalization group equations for soft supersymmetry breaking couplings, Phys. Rev. D 50 (1994) 2282 [Erratum ibid. D 78 (2008) 039903] [hep-ph/9311340] [INSPIRE].

[88] K. Benakli, M.D. Goodsell and A.-K. Maier, Generating mu and Bmu in models with Dirac Gauginos, Nucl. Phys. B 851 (2011) 445 [arXiv:1104.2695] [INSPIRE]. 
[89] N. Arkani-Hamed, A. Gupta, D.E. Kaplan, N. Weiner and T. Zorawski, Simply Unnatural Supersymmetry, arXiv:1212.6971 [INSPIRE].

[90] H. Cheng, D. Kaplan, M. Schmaltz and W. Skiba, Deconstructing gaugino mediation, Phys. Lett. B 515 (2001) 395 [hep-ph/0106098] [INSPIRE].

[91] C. Csáki, J. Erlich, C. Grojean and G.D. Kribs, $4-D$ constructions of supersymmetric extra dimensions and gaugino mediation, Phys. Rev. D 65 (2002) 015003 [hep-ph/0106044] [INSPIRE].

[92] A. De Simone, J. Fan, M. Schmaltz and W. Skiba, Low-scale gaugino mediation, lots of leptons at the LHC, Phys. Rev. D 78 (2008) 095010 [arXiv:0808.2052] [INSPIRE].

[93] F. Mescia and J. Virto, Natural SUSY and Kaon Mixing in view of recent results from Lattice QCD, Phys. Rev. D 86 (2012) 095004 [arXiv:1208.0534] [InSPIRE].

[94] G.F. Giudice, M. Nardecchia and A. Romanino, Hierarchical Soft Terms and Flavor Physics, Nucl. Phys. B $\mathbf{8 1 3}$ (2009) 156 [arXiv:0812.3610] [InSPIRE].

[95] R. Barbieri, E. Bertuzzo, M. Farina, P. Lodone and D. Pappadopulo, A Non Standard Supersymmetric Spectrum, JHEP 08 (2010) 024 [arXiv: 1004.2256] [INSPIRE].

[96] A. Crivellin et al., SUSY_FLAVOR v2: A Computational tool for FCNC and CP-violating processes in the MSSM, Comput. Phys. Commun. 184 (2013) 1004 [arXiv:1203.5023] [INSPIRE].

[97] L.J. Hall and Y. Nomura, Gauge unification in higher dimensions, Phys. Rev. D 64 (2001) 055003 [hep-ph/0103125] [INSPIRE].

[98] G. Durieux and C. Smith, The same-sign top signature of R-parity violation, JHEP 10 (2013) 068 [arXiv: 1307.1355] [InSPIRE].

[99] J. Berger, M. Perelstein, M. Saelim and P. Tanedo, The Same-Sign Dilepton Signature of RPV/MFV SUSY, JHEP 04 (2013) 077 [arXiv: 1302.2146] [INSPIRE].

[100] J. Alwall, M. Herquet, F. Maltoni, O. Mattelaer and T. Stelzer, MadGraph 5 : Going Beyond, JHEP 06 (2011) 128 [arXiv: 1106. 0522] [INSPIRE].

[101] B. Allanach, SOFTSUSY: a program for calculating supersymmetric spectra, Comput. Phys. Commun. 143 (2002) 305 [hep-ph/0104145] [INSPIRE].

[102] P. Meade and M. Reece, BRIDGE: Branching ratio inquiry/decay generated events, hep-ph/0703031 [inSPIRE].

[103] M. Muhlleitner, A. Djouadi and Y. Mambrini, SDECAY: A Fortran code for the decays of the supersymmetric particles in the MSSM, Comput. Phys. Commun. 168 (2005) 46 [hep-ph/0311167] [INSPIRE].

[104] W. Beenakker, R. Hopker and M. Spira, PROSPINO: A Program for the production of supersymmetric particles in next-to-leading order QCD, hep-ph/9611232 [INSPIRE].

[105] T. Sjöstrand, S. Mrenna and P.Z. Skands, A Brief Introduction to PYTHIA 8.1, Comput. Phys. Commun. 178 (2008) 852 [arXiv:0710.3820] [InSPIRE].

[106] S. Ovyn, X. Rouby and V. Lemaitre, DELPHES, a framework for fast simulation of a generic collider experiment, arXiv:0903.2225 [INSPIRE]. 